Electronic Journal of Statistics

Vol. 15 (2021) 4868-4915

ISSN: 1935-7524

https://doi.org/10.1214/21-EJS1892

\title{
Estimating high-dimensional covariance and precision matrices under general missing dependence*
}

\author{
Seongoh Park \\ Department of Statistics, \\ Sungshin Women's University, \\ Seoul, Korea \\ e-mail: spark6@sungshin.ac.kr \\ Xinlei Wang \\ Department of Statistical Science, \\ Southern Methodist University, \\ Dallas, TX, USA \\ e-mail: swang@smu.edu \\ Johan $\operatorname{Lim}^{\dagger}$ \\ Department of Statistics, \\ Seoul National University, \\ Seoul, Korea \\ e-mail: johanlim@snu.ac.kr
}

\begin{abstract}
A sample covariance matrix $\boldsymbol{S}$ of completely observed data is the key statistic in a large variety of multivariate statistical procedures, such as structured covariance/precision matrix estimation, principal component analysis, and testing of equality of mean vectors. However, when the data are partially observed, the sample covariance matrix from the available data is biased and does not provide valid multivariate procedures. To correct the bias, a simple adjustment method called inverse probability weighting (IPW) has been used in previous research, yielding the IPW estimator. The estimator can play the role of $\boldsymbol{S}$ in the missing data context, thus replacing $\boldsymbol{S}$ in off-the-shelf multivariate procedures such as the graphical lasso algorithm. However, theoretical properties (e.g. concentration) of the IPW estimator have been only established in earlier work under very simple missing structures; every variable of each sample is independently subject to missingness with equal probability. We investigate the deviation of the IPW estimator when observations are partially observed under general missing dependency. We prove the optimal convergence rate $O_{p}(\sqrt{\log p / n})$ of the IPW estimator based on the element-wise maximum norm, even when two unrealistic assumptions (known mean and/or missing probabilities) frequently assumed to be known in the past work are relaxed. The optimal rate is especially crucial in estimating a precision matrix, because of the "meta-theorem" [26] that claims the rate of the IPW estimator governs that of the resulting precision matrix estimator. In
\end{abstract}

*Seongoh Park is supported by the Sungshin Women's University Research Grant of H20210143, Johan Lim is supported by the National Research Foundation of Korea (NRF) grant funded by the Korea government (MSIT) (NRF-2017R1A2B2012264).

${ }^{\dagger}$ Corresponding author. 
the simulation study, we discuss one of practically important issues, nonpositive semi-definiteness of the IPW estimator, and compare the estimator with imputation methods.

MSC2020 subject classifications: Primary 62H12; secondary 60E15.

Keywords and phrases: Convergence rate, covariance matrix, dependent missing structure, element-wise maximum norm, inverse probability weighting.

Received April 2021.

\section{Contents}

1 Introduction . . . . . . . . . . . . . . . . . . . . . . . 4870

1.1 Existing work on (inverse) covariance matrix estimation with missing values . . . . . . . . . . . . . . . . . . 4870

1.2 Our contributions . . . . . . . . . . . . . . . . . . . . 4871

1.3 Outline .......................... 4871

2 The IPW estimator under general missing dependency and its rate . 4872

2.1 Notation . . . . . . . . . . . . . . . . . . . . . . . 4874

2.2 Main results . . . . . . . . . . . . . . . . . . . . . 4874

2.3 The meta-theorem in estimation of a precision matrix . . . . 4877

3 Relaxation of implicit assumptions . . . . . . . . . . . . . . . . . 4879

3.1 The case of unknown mean . . . . . . . . . . . . . . . . 4879

3.2 The case of unknown missing probability . . . . . . . . . 4881

4 Non-positive semi-definiteness of the plug-in estimator . . . . . . . . 4882

5 Numerical study . . . . . . . . . . . . . . . . . . . . . . . . . 4884

5.1 Setting . . . . . . . . . . . . . . . . . . 4884

5.2 The rate of convergence . . . . . . . . . . . . 4885

5.3 Comparison with imputation methods . . . . . . . . . . 4885

5.4 Unknown mean . . . . . . . . . . . . . . . . . . . . . 4886

6 Application to real data . . . . . . . . . . . . . . . 4888

7 Discussion . . . . . . . . . . . . . . . . . . . . . . . . . . 4888

A Auxiliary lemmas . . . . . . . . . . . . . . . . . . . . . 4889

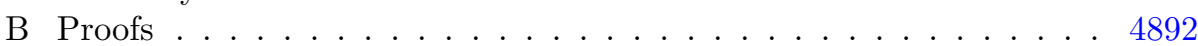

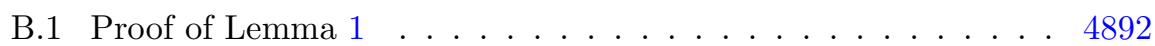

B.2 Proof of Theorem 1. . . . . . . . . . . . . . . . . 4894

B.3 Proof of Corollary $2 \ldots \ldots \ldots$. . . . . . . . . . . 4894

B.4 Proof of Theorem 2 . . . . . . . . . . . . . . . . 4896

B.5 Proof of Lemma $5 \ldots \ldots \ldots$

B.6 Proof of Theorem 3. . . . . . . . . . . . . . . . . 4900

C Additional analyses and details in Section $4 \ldots \ldots$. . . . . . . 4901

C.1 Non-PSD input for CLIME . . . . . . . . . . . . . . . . . 4901

C.2 Failure of Algorithm 1 under missing data . . . . . . . . . . 4902

D Additional analyses and details in Section 5 . . . . . . . . . . 4903

D.1 Details of the simulation setting . . . . . . . . . . . . 4903

D.2 Additional results of the simulation study . . . . . . . . 4905 
E Details in Section $6 \ldots \ldots$. . . . . . . . . . . . . . . 4907

Acknowledgments . . . . . . . . . . . . . . . . . . . . . . . 4911

References . . . . . . . . . . . . . . . . . . . . . 4911

\section{Introduction}

One of the overarching themes in statistical and machine learning societies is to discover complex relationships among high-dimensional variables. Out of many approaches to understand dependency among variables, the covariance matrix and its inverse matrix (i.e., the precision matrix) are arguably important statistical tools in this line of research. Hence, methodological and theoretical analyses of these statistics, such as scalability, consistency, and convergence rate, have been established by many researchers (see the section Introduction from [11] for a comprehensive literature review, and references therein), because of their utility in a broad range of disciplines such as biology, geophysics, economics, public health, and social sciences. Despite much advance over decades in the estimation of a covariance/precision matrix under the high-dimensional setting, most approaches to date have been oblivious to handling missing observations. However, widespread applications have emerged in modern sciences where the primary interest is placed on estimating the correlation structure from observations subject to missingness. To name a few, climate data ([40]), genomic studies $([8,24])$, and remote sensing data $([14])$. Even so, there has been relatively less development in both methodology and theory that deal with the (inverse) covariance estimation problem in the presence of missing data.

\subsection{Existing work on (inverse) covariance matrix estimation with missing values}

Previous research in the field of estimation of an (inverse) covariance matrix with incomplete data, though not many to our best knowledge, can be classified into two branches; the likelihood-based approach and the plug-in approach.

The first line of the works is the likelihood-based inference, mostly achieving the maximum likelihood estimator by an expectation-maximization (EM) algorithm (or its variants) $([19,41,1,42,24])$. In spite of individual successes in covariance/precision matrix estimation when missing observations are present, the major drawback of this approach is separate development of estimating algorithms and supporting theories. That is, one considering a new proposal under this framework should put huge efforts on implementing the new method for practical purposes and prove theoretical properties (e.g. consistency). Furthermore, the Gaussian assumption on observations commonly used in the likelihood inference could be restrictive in the high-dimensional setting.

The other scheme of research studied rather in recent years is based on the fact that many procedures for estimating a covariance/precision matrix solely rely on the sample covariance matrix $\boldsymbol{S}$, not the data itself. However, if missing observations exist, the sample covariance matrix $\boldsymbol{S}_{Y}$ (see the definition in 
(1)) using partial observations is no longer a proper estimator. Thus, preceding work ([29, 23, 7, 46, 34, 33]) have considered adjusting the missing proportion, or a bias that appears in $\boldsymbol{S}_{Y}$. The modified estimator is often referred to as an inverse probability weighting (IPW) estimator and is plugged in procedures of multivariate data analyses instead of $\boldsymbol{S}_{Y}$. For example, [23] put the IPW estimator into the graphical lasso algorithm ([13]) to estimate a sparse precision matrix, while [7] plug it in banding, tapering, or thresholding operators to recover a structured covariance matrix in the missing data context. [46] apply the CLIME method ([5]) to the bias-corrected rank-based correlation matrix to estimate a sparse precision matrix of a non-paranormal distribution. In the low-rank approximation problem, the IPW estimator is plugged into the matrix lasso ([36]) by [29], which is extended by [34] to vector autoregressive processes. All of these works are based on one common assumption about missingness; for each sample, each variable is independently subject to missingness with equal (uniform) probability. Their theoretical analyses, though recovering the aimed rate $\sqrt{\log p / n}$ ( $n$ : the sample size, $p$ : dimension), are established based on such a restrictive independence assumption. In contrast, dependent (and non-uniform) missing structure has not been paid attention to until very recent year when [31] made an initial attempt and [33] made a further investigation. While those papers are based on the spectral norm using the effective rank of a matrix (see Table 1), this paper derive the optimal convergence rate of the IPW estimator in terms of the element-wise maximum norm under general missing dependency.

\subsection{Our contributions}

Our main contributions are outlined below.

Derivation of the optimal convergence rate under dependent missing structure. We develop a non-asymptotic deviation inequality of the IPW estimator in the element-wise maximum norm by extending missing independence to missing dependency (Theorem 1). The theoretical results maintain the conventional convergence rate $\sqrt{\log p / n}$ achieved by the earlier works ([3]) and the references in Table 1). Theorem 1 can be further incorporated with existing theories in estimation of structured precision matrix, because assumptions made in this paper do not conflict with those made in the existing theories.

Relaxation of implicit assumptions to derive the rates. In analyzing the concentration of the IPW estimator, estimation of the population mean and missing probabilities has been largely unexplored ([29], [46], [31], [33]), which is not desirable in practice. Filling the gaps, this paper establishes the concentration inequalities for the IPW estimator under unknown mean (Theorem 2) and missing probabilities (Theorem 3).

\subsection{Outline}

The remainder of this paper is organized as follows. At the beginning of Section 2 , we formally state the problem setup and introduce the IPW estimator under 
general missing dependency. Under the setting where the missing probabilities and population means are assumed to be known, we present our theoretical results related to the estimator in Section 2 and apply them to estimation of a sparse precision matrix. In Section 3, the two unrealistic assumptions are relaxed, and we show similar results to the previous section. Section 4 deals with non-positive semi-definiteness of the IPW estimator and its potential remedies. In Section 5 and 6, we show our numerical studies on simulated and real data, respectively. We conclude this paper with a brief discussion and summary in Section 7.

\section{The IPW estimator under general missing dependency and its rate}

Let $X=\left(X_{1}, \ldots, X_{p}\right)^{\mathrm{T}}$ be a $p$-dimensional vector of random variables with mean zero and covariance matrix $\boldsymbol{\Sigma}=\mathbb{E}\left(X X^{\mathrm{T}}\right)$. We denote missing observations by 0 , which has a simple mathematical representation using a missing indicator ${ }^{1}$ $\delta_{j}$ that takes its value either 0 (missing) or 1 (observed);

$$
Y=\left(Y_{1}, \ldots, Y_{p}\right)^{\mathrm{T}}, \quad Y_{j}=\delta_{j} X_{j}, \quad j=1, \ldots, p .
$$

The multivariate binary vector $\delta=\left(\delta_{1}, \ldots, \delta_{p}\right)^{\mathrm{T}}$ is assumed to follow some distribution where a marginal distribution of $\delta_{j}$ is the Bernoulli distribution with success probability $0 \leq \pi_{j} \leq 1$. This formulation is an extension of independent missing structure used in previous works $([29,46,23,6])$, which assume $\delta_{k}$ is independent of $\delta_{\ell}(k \neq \ell)$. Contrary to it, this paper assumes the $p$ random variables $\left\{\delta_{j}, j=1, \ldots, p\right\}$ are allowed to be dependent and not identically distributed. The probability of observing at multiple positions is henceforth denoted by

$$
\mathrm{P}\left(\delta_{i}=\delta_{j}=\delta_{k}=\ldots=1\right)=\pi_{i j k \ldots} .
$$

Dependent missing structure naturally occurs through a longitudinal clinical study since a patient absent at visit (=variable) $k$ would have more possibility of not showing up at forthcoming visits $\ell(>k)$. There exists more general and plausible scenarios where extrinsic covariates are involved in occurrence of missingness.

Let us consider $n$ samples from the population above where the covariance matrix $\boldsymbol{\Sigma}=\left(\sigma_{k \ell}, 1 \leq k, \ell \leq p\right)$ is to be estimated. Denote the $i$-th sample version of $X, Y, \delta_{j}$ by $X_{i}, Y_{i}, \delta_{i j}$, respectively. Then, the sample covariance matrix from partially observed data is obtained by

$$
\boldsymbol{S}_{Y}=\frac{1}{n} \sum_{i=1}^{n} Y_{i} Y_{i}^{\mathrm{T}}=\left(\frac{1}{n} \sum_{i=1}^{n} \delta_{i j} \delta_{i k} X_{i j} X_{i k}, 1 \leq j, k \leq p\right)
$$

\footnotetext{
${ }^{1}$ Technically, this is a "response" indicator as termed in [22], since the value 1 indicates an observed (responded) variable, but we insist on using "missing" to emphasize the context of missing data.
} 
It can be easily checked that $\boldsymbol{S}_{Y}$ is biased for $\boldsymbol{\Sigma}$, since its expectation is $\boldsymbol{\Sigma}^{\pi}=$ $\left(\pi_{j k} \sigma_{j k}, 1 \leq j, k \leq p\right)$ by assuming independence between $\left\{X_{i}\right\}_{i=1}^{n}$ and $\left\{\delta_{i j}\right\}_{i, j}$. This motivates one to adjust each component of $\boldsymbol{S}_{Y}$ by a weight and define the IPW estimator $\widehat{\boldsymbol{\Sigma}}^{I P W}=\left(\left(\widehat{\boldsymbol{\Sigma}}^{I P W}\right)_{j k}, 1 \leq j, k \leq p\right)$ by

$$
\left(\widehat{\boldsymbol{\Sigma}}^{I P W}\right)_{j k}= \begin{cases}\frac{1}{n} \sum_{i=1}^{n} \frac{\delta_{i j}}{\pi_{j}} X_{i j}^{2} & j=k, \\ \frac{1}{n} \sum_{i=1}^{n} \frac{\delta_{i j} \delta_{i k}}{\pi_{j k}} X_{i j} X_{i k} & j \neq k,\end{cases}
$$

provided that $\pi_{j k}>0, \forall j, k$. Then, $\widehat{\boldsymbol{\Sigma}}^{I P W}$ is unbiased for $\boldsymbol{\Sigma}$ under the missing completely at random (MCAR) mechanism ([25]), that is, $\left\{\delta_{i j}\right\}_{j=1}^{p}$ is independent of $\left\{X_{i j}\right\}_{j=1}^{p}$ for $i=1, \ldots, n$. For example, when data acquisition is carried out through censors (e.g. remote sensing data), loss of data arises due to faults in censors and thus is independent of values to be measured.

We note this adjustment technique is frequently used in a general context of missing data and also known as the propensity score method. The underlying idea of it is to construct an unbiased estimating equation by reweighting the contribution of each sample on the equation. The corresponding equation for the covariance estimation problem under the Gaussian setting without missingness is a score function given by

$$
\frac{1}{n} \sum_{i=1}^{n} Q\left(X_{i} ; \mathbf{\Sigma}\right)=0
$$

where $Q\left(X_{i} ; \boldsymbol{\Sigma}\right)=\boldsymbol{\Sigma}^{-1} X_{i} X_{i}^{\mathrm{T}} \boldsymbol{\Sigma}^{-1}-\boldsymbol{\Sigma}^{-1}$. Since (3) is equivalent to solving $n^{-1} \sum_{i=1}^{n}\left(X_{i} X_{i}^{\mathrm{T}}-\boldsymbol{\Sigma}\right)=0$, the reweighted version of the equation above would be

$$
\frac{1}{n} \sum_{i=1}^{n} \boldsymbol{R}_{i} *\left(X_{i} X_{i}^{\mathrm{T}}-\boldsymbol{\Sigma}\right)=0
$$

where $\boldsymbol{R}_{i}=\left(\delta_{i j} \delta_{i k} / \pi_{j k}, 1 \leq j, k \leq p\right)$ and $*$ is an element-wise product. Solving the equation above with respect to $\boldsymbol{\Sigma}$ yields an empirical version of the IPW estimator that replaces $\pi_{j k}$ in (2) with $n^{-1} \sum_{i=1}^{n} \delta_{i j} \delta_{i k}$. This estimator has been used and analyzed before in [23] and [7], which will be studied in Section 3.2 of this paper under general missing dependency. Remark that the inverse probability $\pi_{j k}$ in $\boldsymbol{R}_{i}$ is ignorable and does not play any role in defining the empirical estimator. However, when the probability is dependent on sample-specific variables $\left(X_{i}\right.$ or extrinsic covariates $\left.W_{i}\right)$, we should give weights in the form of the conditional probability defined by $\mathrm{P}\left(\delta_{i j}=\delta_{i k}=1 \mid X_{i}, W_{i}\right)$, which adjusts the selection bias from partial observations $\left\{i: \delta_{i j}=\delta_{i k}=1\right\}$. For the sake of simplicity, analyses in this paper only concern the identical setting on missing indicators, that is, $\pi_{j k \ell \ldots} \stackrel{\forall i}{=} \mathrm{P}\left(\delta_{i j}=\delta_{i k}=\delta_{i \ell}=\ldots=1\right)$. 


\subsection{Notation}

Throughout this paper, we use the following matrix norms; for a matrix $\boldsymbol{A}$, the element-wise maximum norm is $\|\boldsymbol{A}\|_{\max }=\max _{i, j}\left|\boldsymbol{A}_{i j}\right|$, the operator 1-norm is $\|\boldsymbol{A}\|_{1}=\max _{j} \sum_{i}\left|\boldsymbol{A}_{i j}\right|$, the operator 2-norm $\|\boldsymbol{A}\|_{2}$ is the largest singular value (or eigenvalue if $\boldsymbol{A}$ is symmetric), the element-wise 1-norm is $|\boldsymbol{A}|_{1}=\sum_{i, j}\left|\boldsymbol{A}_{i j}\right|$, and the Frobenius norm is $\|\boldsymbol{A}\|_{F}=\sqrt{\sum_{i, j} \boldsymbol{A}_{i j}^{2}} \cdot \operatorname{diag}(\boldsymbol{A})$ is a diagonal matrix whose diagonal entries are inherited from $\boldsymbol{A}$. For a vector $v$, we define $\|v\|_{1}=$ $\sum_{j}\left|v_{j}\right|$. Also, we define $R(\theta)=\exp \left(1 /\left(4 e \theta^{2}\right)\right)-1 / 2-1 /\left(4 e \theta^{2}\right)$ for $\theta>0$, which is monotonically decreasing and satisfies $R(\theta)>1 / 2$.

\subsection{Main results}

We state our assumptions used in the following theoretical analyses; (i) subGaussianity for each component of $X_{i}$, (ii) a general dependency structure for $\delta_{i}$, and (iii) MCAR for missing mechanism. We begin with one of the equivalent definitions of the sub-Gaussian variable ([44]): the uniformly bounded moments.

Assumption 1 (Sub-Gaussianity). $X$ is a sub-Gaussian random variable in $\mathbb{R}$ satisfying

$$
\mathbb{E} X=0, \quad \mathbb{E} X^{2}=1, \quad \text { and } \sup _{r \geq 1} \frac{\left\{\mathbb{E}|X|^{r}\right\}^{1 / r}}{\sqrt{r}} \leq K
$$

for some $K>0$.

We note that the Gaussian random variable $X \sim N\left(0, \sigma^{2}\right)$ satisfies

$$
\sup _{r \geq 1}\left\{\mathbb{E}|X|^{r}\right\}^{1 / r} / \sqrt{r} \leq \sigma K
$$

for some numeric constant $K>0$. Missing is assumed to occur with general dependency in sense of the following;

Assumption 2 (General missing dependency). A missing indicator vector $\delta=\left(\delta_{1}, \ldots, \delta_{p}\right)^{\mathrm{T}} \in\{0,1\}^{p}$ follows some multivariate distribution where each marginal distribution is a Bernoulli distribution with a missing probability ${ }^{2}$ $\pi_{j} \in(0,1]$, i.e., $\delta_{j} \sim \operatorname{Ber}\left(\pi_{j}\right)$. Further, assume that $\pi_{j k} \neq 0$ for all $1 \leq j, k \leq p$.

This distribution is examined by [9] where they call it the multivariate Bernoulli distribution. If interaction terms are considered up to the secondorder, this multivariate model coincides with a well-known Ising model. The non-degenerate condition for the missing probabilities (i.e., $\pi_{j}>0, \pi_{j k}>0$ ) is required since, for example, $\pi_{j k}=0$ implies no data could be observed for estimating the second moment $\sigma_{j k}$, which is unrealistic for our discussion. Next, we formally state our missing mechanism again;

\footnotetext{
${ }^{2}$ Following the previous footnote, this is called a "missing" probability.
} 
Assumption 3 (Missing completely at random). An event that an observation is missing is independent of both observed and unobserved random variables.

Under the data structure in this paper, the above mechanism essentially says that two random vectors, $\delta_{i}$ and $X_{i}$, are independent. We note that Assumptions 1 and 3 are commonly used in the context of covariance estimation with incomplete data, while Assumption 2 is more general than the independent structure that previous research depends on. Based on these assumptions, Lemma 1 describes the element-wise deviation of the IPW estimator from a true covariance matrix.

Lemma 1. Let $\left\{X_{i}\right\}_{i=1}^{n}$ be i.i.d. random vectors in $\mathbb{R}^{p}$ with mean 0 and covariance $\boldsymbol{\Sigma}$. Suppose the scaled random variable $X_{i k} / \sqrt{\sigma_{k k}}$ satisfies Assumption 1 with a constant $K>0$ for all $k$. Also, let $\left\{\delta_{i}\right\}_{i=1}^{n}$ be i.i.d. binary random vectors satisfying Assumption 2. By observing samples $\left\{Y_{i}\right\}_{i=1}^{n}$ under Assumption 3, we have

$$
\mathrm{P}\left[n^{-1}\left|\sum_{i=1}^{n}\left(\frac{Y_{i k} Y_{i \ell}}{\pi_{k \ell}}-\sigma_{k \ell}\right)\right| \geq \frac{C\left(\sigma_{k k} \sigma_{\ell \ell}\right)^{1 / 2} K^{2} R(K)^{1 / 2}}{\pi_{k \ell}} t\right] \leq 4 \exp \left(-n t^{2}\right),
$$

if $t \geq 0$ satisfies

$$
\begin{cases}t^{2} \leq c R\left(\frac{2 K}{\sqrt{\pi_{k}+\pi_{\ell}-2 \pi_{k \ell}\left|\rho_{k \ell}\right|}}\right), & \text { if } k \neq \ell, \\ t^{2} \leq c R\left(K / \sqrt{\pi_{k}}\right), & \text { if } k=\ell\end{cases}
$$

where $c, C>0$ are scalar constants and $\rho_{k \ell}=\sigma_{k \ell} / \sqrt{\sigma_{k k} \sigma_{\ell \ell}}$.

A proof of Lemma 1 can be found in Section B.1 of Appendix. We provide some remarks as regards this lemma. This concentration inequality covers the existing results as special cases. First, if data is assumed to be fully observed (i.e., $\pi_{k \ell}=1, \forall k, \ell$ ), then (6) is reduced to

$$
\mathrm{P}\left[n^{-1}\left|\sum_{i=1}^{n}\left(X_{i k} X_{i \ell}-\sigma_{k \ell}\right)\right| \geq C_{1} \sqrt{\sigma_{k k} \sigma_{\ell \ell}} t\right] \leq C_{2} \exp \left(-n t^{2}\right), \quad 0 \leq t \leq C_{3},
$$

where $C_{1}, C_{2}, C_{3}$ are scalar constants. It can be seen that this form is equivalent to Lemma A.3. in [4] (Gaussian) or Lemma 1 in [35] (sub-Gaussian), up to multiple constant difference. When an independent and identical structure of missing indicators is assumed (i.e., $\delta_{k} \stackrel{\forall k}{\sim} \operatorname{Ber}(\pi)$ ) in Lemma 1, the reduced probabilistic bound is similar to that from [23] (plugging in $t \leftarrow \sqrt{\log (4 / \delta) / n}$ in (6))

$$
\mathrm{P}\left[n^{-1}\left|\sum_{i=1}^{n}\left(\frac{Y_{i k} Y_{i \ell}}{\pi^{2}}-\sigma_{k \ell}\right)\right| \geq \frac{C K^{2}}{\pi^{2}} \sqrt{\frac{R(K) \sigma_{k k} \sigma_{\ell \ell} \log (4 / \delta)}{n}}\right] \leq \delta
$$

for the sample size $n$ chosen according to Lemma 1. Rigorously speaking, the proposed IPW estimator in Lemma 1 and that of [23] (see (11)) are different 
by the inverse weighting factor when correcting missing observations. However, replacing missing probabilities with unbiased empirical estimates will not cause a considerable change in our result (see Section 3.2).

Using the lemma above, the rate of convergence of the IPW estimator can be derived in terms of the element-wise maximum norm. Let us define the maximum and minimum value of parameters that appear in Lemma 1 as follows;

$$
\sigma_{\max }=\max _{k} \sigma_{k k}, \quad \pi_{\min }=\min _{k, \ell} \pi_{k \ell}, \quad v_{\min }=\min _{k \neq \ell}\left(\pi_{k}+\pi_{\ell}-2 \pi_{k \ell}\left|\rho_{k \ell}\right|\right) .
$$

Theorem 1. Assume the conditions of Lemma 1 hold, and further assume the sample size and dimension satisfy

$$
n / \log p>c_{1} / R\left(2 K / \sqrt{v_{\min }}\right)
$$

then

$$
\mathrm{P}\left[\left\|\widehat{\boldsymbol{\Sigma}}^{I P W}-\boldsymbol{\Sigma}\right\|_{\max } \geq \frac{C_{1} \sigma_{\max } K^{2}}{\pi_{\min }} \sqrt{\frac{R(K) \log p}{n}}\right] \leq 4 p^{-1},
$$

where $c_{1}, C_{1}>0$ are scalars.

A proof of the theorem can be found in Section B.2 of Appendix.

The above result provides a few intuitions. First of all, the convergence rate $\sqrt{\log p / n}$ is satisfied with the IPW estimator when missing data are present. Also, small portion of missingness in data agrees with a faster convergence rate since $\pi_{\min } \approx 1$. Furthermore, if we reparametrize the missing probabilities by $\left\{p_{k \ell}^{(a, b)}: a, b=0,1\right\}$ where $p_{k \ell}^{(a, b)}=\mathrm{P}\left(\delta_{i k}=a, \delta_{i \ell}=b\right)$, then we see that the entries in $v_{\min }$ are rewritten by

$$
\pi_{k}+\pi_{\ell}-2 \pi_{k \ell}\left|\rho_{k \ell}\right|=p_{k \ell}^{(1,0)}+p_{k \ell}^{(0,1)}+2 p_{k \ell}^{(1,1)}\left(1-\left|\rho_{12}\right|\right), \quad k, \ell=1, \ldots, p .
$$

Thus, if less observations are missing (i.e. larger values of $p_{k \ell}^{(1,0)}, p_{k \ell}^{(0,1)}, p_{k \ell}^{(1,1)}$ ), less samples are needed to achieve the same convergence rate.

If we assume an independent structure on missing indicators, we get the following result, which is comparable to those from [23], [29], and [32]. Let $\widehat{\boldsymbol{\Sigma}}_{i n d}^{I P W}$ be the IPW estimator (2) with $\pi_{j k}=\pi^{2}, j \neq k$ and $\pi_{j j}=\pi$ for all $j, k$.

Corollary 1 (Identical and independent missing structure). Under the conditions of Lemma 1, we further assume $\delta_{i k} \sim \operatorname{Ber}(\pi)$, independently, $k=1, \ldots, p$. Then, when the sample size and dimension satisfy

$$
n / \log p>c_{1}\left\{\exp \left(\frac{\pi\left(1-\pi \rho_{\max }\right)}{8 e K^{2}}\right)-\frac{1}{2}-\frac{\pi\left(1-\pi \rho_{\max }\right)}{8 e K^{2}}\right\}^{-1}
$$

then

$$
\mathrm{P}\left[\left\|\widehat{\boldsymbol{\Sigma}}_{\text {ind }}^{I P W}-\boldsymbol{\Sigma}\right\|_{\max } \geq \frac{C_{1} \sigma_{\max } K^{2}}{\pi^{2}} \sqrt{\frac{R(K) \log p}{n}}\right] \leq 4 p^{-1},
$$

where $c_{1}, C_{1}>0$ are scalrs and $\rho_{\max }=\max _{k \neq \ell}\left|\rho_{k \ell}\right|$. 
First, we note [32] prove a bound with general element-wise $q$-norm under non-uniform missing probabilities (i.e., $\pi_{j k}=\pi_{j} \pi_{k}, j \neq k$ ). By sending $q \rightarrow \infty$ in Theorem 1 of [32], we could obtain a deviation inequality in the element-wise maximum norm, which is given in Table 1 and comparable to Corollary 1 .

Second, for comparison with other previous research $([23,29,46])$, we observe that the Taylor expansion of an exponential function yields

$\left\{\exp \left(\frac{\pi\left(1-\pi \rho_{\max }\right)}{8 e K^{2}}\right)-\frac{1}{2}-\frac{\pi\left(1-\pi \rho_{\max }\right)}{8 e K^{2}}\right\}^{-1}=b_{1} /\left(1+b_{2} \pi^{2}+o\left(\pi^{2}\right)\right)$, as $\pi \rightarrow 0$,

for some scalars $b_{1}, b_{2}>0$. Therefore, the sample size (relative to the dimension) required for accurate estimation is less sensitive to the missing probabilities $\pi$ compared to the previous work whose magnitude is in order of $1 / \pi^{2}$ (see Table 1). However, the bound of the IPW estimator in the element-wise maximum norm increases in the order of magnitude $1 / \pi^{2}$, which is larger than the rate $1 / \pi$ claimed in other literature (see Table 1 ).

Finally, Table 1 summarizes the rate and sample size of the IPW estimator from the related works. [7] have considered the minimax optimality (with a structured covariance matrix), which is, however, not comparable to what is given in Table 1. Hence, their work is not included here.

Table 1 shows the rate of convergence $\sqrt{\log p / n}$ has appeared in the previous literature. When dependency for missing indicators is allowed, the achieved rate in [31] under the spectral norm is not optimal, though they have first tackled it. Very recently, [33] show an improved rate for expectation of an estimation error based on the spectral norm. In terms of the element-wise maximum norm, to our best knowledge, this paper is among the first to obtain the optimal rate.

\subsection{The meta-theorem in estimation of a precision matrix}

The derived concentration inequality is crucial because of its application to precision matrix estimation. The related theory, known as the meta-theorem that has first appeared in [26], implies that the rate of the precision matrix estimator $\widehat{\Omega}$ is determined by the rate $\|\cdot\|_{\max }$ of an input matrix (e.g. the IPW estimator) used to estimate $\widehat{\boldsymbol{\Omega}}$. Therefore, when there is no missing observation, the success of the graphical lasso ([35]), the CLIME ([5]), and the graphical Dantzig selector $([48])$ in accurate estimation and graph recovery depends on the fact that the sample covariance matrix $\boldsymbol{S}$ satisfies

$$
\mathrm{P}\left(\|\boldsymbol{S}-\boldsymbol{\Sigma}\|_{\max } \geq C \sqrt{\log p / n}\right) \leq d / p,
$$

for some $C, d>0$. To grasp the underlying mechanism of the meta-theorem, we refer readers to the proof of Corollary 2. Since the claimed rate of convergence in Theorem 1 is the same as that of $\boldsymbol{S}$ in (8), the meta-theorem also guarantees the same optimal rates of the precision matrix estimators with missing observations.

It should be remarked that the rate in Theorem 1 is not driven for a certain class of covariance/precision matrices (e.g. sparse or low-rank) or with a specific 
TABLE 1. Summary of literature using the idea of the IPW estimator. $M=\left(1 / \pi_{k \ell}, 1 \leq k, \ell \leq p\right) . \sigma_{\max }=\max _{k} \sigma_{k k}, \pi_{\min }=\min _{k, \ell} \pi_{k \ell}$. "Rate" is the convergence rate (up to a constant factor depending only on distributional parameters) of an estimator ("Est.") measured by a matrix norm ("Norm"). "Assump." indicates which structure of missing dependency is imposed to derive the rate. "Size" is a condition for $n$ and $p$ to guarantee the rate holds with probability at least $1 / p$. In the first column, we use the following labels: L2014=[29], KX2012=[23], W2014=[46], PL2019=[31], PO2018=[32], and PO2019=[33].

\begin{tabular}{|c|c|c|c|c|c|}
\hline Article & Est. & Assump. & Norm & Rate & Size \\
\hline K2012 & $\widehat{\boldsymbol{\Sigma}}^{e m p}(11)$ & Indep & $\|\cdot\|_{\max }$ & $\sigma_{\max } \sqrt{\frac{\log (8 p)}{\pi^{2} n-\sqrt{2 \pi^{2} n \log (2 p)}}}$ & $p=O\left(\exp \left(n \pi^{2}\right)\right)$ \\
\hline L2014 & $\widehat{\boldsymbol{\Sigma}}_{\text {ind }}^{I P W}$ & Indep & $\|\cdot\|_{2}$ & $\sqrt{\frac{\operatorname{tr}(\boldsymbol{\Sigma})\|\boldsymbol{\Sigma}\|_{2} \log p}{\pi^{2} n}}$ & $p=O\left(\exp \left(\frac{n \pi^{2}\|\boldsymbol{\Sigma}\|_{2}}{\operatorname{tr}(\boldsymbol{\Sigma})}\right)\right)$ \\
\hline PO2018 & $\widehat{\boldsymbol{\Sigma}}_{i n d}^{I P W}$ & Indep & $\|\cdot\|_{\max }$ & $\sigma_{\max } \sqrt{\frac{\log p}{\min _{k} \pi_{k}^{4} n}}$ & $p=O(\exp (n))$ \\
\hline W2014 & Spearman's $\rho$ & Indep & $\|\cdot\|_{\max }$ & $\sqrt{\frac{\log p}{\pi^{2} n}}$ & $p=O\left(\exp \left(n \pi^{2}\right)\right)$ \\
\hline W2014 & Kendall's $\tau$ & Indep & $\|\cdot\|_{\max }$ & $\sqrt{\frac{\log p}{\pi^{2} n}}$ & $p=O\left(\exp \left(n \pi^{2}\right)\right)$ \\
\hline PL2019 & $\widehat{\boldsymbol{\Sigma}}^{I P W}(2)$ & Depen & $\|\cdot\|_{2}$ & $\|M\|_{2} \sqrt{\frac{\operatorname{tr}(\boldsymbol{\Sigma})\|\boldsymbol{\Sigma}\|_{2} \log p}{n}}$ & $p=O\left(\exp \left(\left\{\frac{n\|\boldsymbol{\Sigma}\|_{2}}{\operatorname{tr}(\boldsymbol{\Sigma})}\right\}^{1 / 3}\right)\right)$ \\
\hline PO2019 & $\widehat{\boldsymbol{\Sigma}}^{I P W}(2)$ & Depen & $\sqrt{\mathbb{E}\|\cdot\|_{2}^{2}}$ & $\sqrt{\frac{\operatorname{tr}(\boldsymbol{\Sigma})\|\boldsymbol{\Sigma}\|_{2} \log p}{\pi_{\min } n}}$ & $p=O\left(\exp \left(\frac{n \pi_{\min }\|\boldsymbol{\Sigma}\|_{2}}{(\log n)^{2} \operatorname{tr}(\boldsymbol{\Sigma})}\right)\right)$ \\
\hline Theorem 1 & $\widehat{\boldsymbol{\Sigma}}^{I P W}(2)$ & Depen & $\|\cdot\|_{\max }$ & $\sigma_{\max } \sqrt{\frac{\log p}{\pi_{\min }^{2} n}}$ & See $(7)$ \\
\hline
\end{tabular}


restriction on $n$ and $p$ such as an asymptotic ratio between them, i.e., $p / n \rightarrow \alpha \in$ $[0, \infty)$. Such flexibility makes it possible to adopt different conditions (on $\boldsymbol{\Sigma}, \boldsymbol{\Omega}$, $n$, or $p$ ) required from different precision matrix estimation methods (e.g. the graphical lasso). We describe the meta-theorem under the dependent missing structure below, which is an extension of Theorem 4.3 in [26]. A proof of the corollary can be found in Section B.3 of Appendix.

Corollary 2. Let the true covariance matrix $\mathbf{\Sigma}$ satisfy the same assumptions that a precision matrix estimation procedure such as the graphical lasso, the graphical Dantzig selector, and the CLIME requires to guarantee the consistency and the support recovery of a graph.

If we plug the IPW estimator $\widehat{\mathbf{\Sigma}}^{I P W}$ into one of the aforementioned methods, the end product retrieves the optimal rate of convergence, and thus has consistency and support recovery properties ${ }^{3}$ even under general missing dependency.

\section{Relaxation of implicit assumptions}

Estimation using the IPW estimator with missing data depends on two implicit assumptions other than Assumptions 1, 2, and 3; known mean (or equivalently zero mean) and missing probabilities. In this section, we will relax such conditions and show corresponding concentration results.

\subsection{The case of unknown mean}

When the first moment of random variables is unknown, an estimator should be modified to be unbiased. It requires a small trick since we do not directly estimate the mean parameter $\mu_{k}$, but $\mu_{k} \mu_{\ell}$ because of the dependent missing structure. The resulting estimator (9) holds the same rate $O_{p}(\sqrt{\log p / n})$ (Theorem 2). It should be pointed out that the extension to the unknown mean case is not so obvious because a quadratic form of sub-Gaussian variables has newly appeared (the second part of (9)). We deal with it by proving a new version of Hanson-Wright inequality described in Lemma 6.

Assume that we observe $\tilde{Y}_{i k}=\delta_{i k} \tilde{X}_{i k}$ where $\tilde{X}_{i k}$ has an unknown mean $\mu_{k}$. Adopting previous notations, we define $X_{i k}$ to satisfy $\tilde{X}_{i k}=X_{i k}+\mu_{k}$. Then, it is easy to show that

$$
\mathbb{E}\left[\sum_{i=1}^{n} \tilde{Y}_{i k} \tilde{Y}_{i \ell}\right]=n \pi_{k \ell}\left(\sigma_{k \ell}+\mu_{k} \mu_{\ell}\right), \quad \mathbb{E}\left[\sum_{i \neq j}^{n} \tilde{Y}_{i k} \tilde{Y}_{j \ell}\right]=n(n-1) \pi_{k} \pi_{\ell} \mu_{k} \mu_{\ell} .
$$

With a simple calculation, we can define the unbiased covariance matrix estimator by $\widehat{\boldsymbol{\Sigma}}^{I P W \mu}=\left(\left(\widehat{\boldsymbol{\Sigma}}^{I P W \mu}\right)_{k \ell}, 1 \leq k, \ell \leq p\right)$ with

$$
\left(\widehat{\boldsymbol{\Sigma}}^{I P W \mu}\right)_{k \ell}=\frac{\sum_{i=1}^{n} \tilde{Y}_{i k} \tilde{Y}_{i \ell}}{n \pi_{k \ell}}-\frac{\sum_{i \neq j}^{n} \tilde{Y}_{i k} \tilde{Y}_{j \ell}}{n(n-1) \pi_{k} \pi_{\ell}} .
$$

\footnotetext{
${ }^{3}$ The support recovery is not guaranteed with the graphical Dantzig selector, since its rate is achieved in the matrix $\ell_{1}$-norm, not $\|\cdot\|_{\max }$.
} 
It is not difficult to find resemblance of (9) with the sample covariance matrix $\boldsymbol{S}$ when data is completely observed. The $(k, \ell)$-th component of $\boldsymbol{S}$ is defined by

$$
\boldsymbol{S}_{k \ell}=\frac{1}{n-1} \sum_{i=1}^{n}\left(\tilde{X}_{i k}-\hat{\mu}_{k}\right)\left(\tilde{X}_{i \ell}-\hat{\mu}_{\ell}\right)
$$

where $\hat{\mu}_{k}=n^{-1} \sum_{i=1}^{n} \tilde{X}_{i k}$, and it can be rearranged by

$$
\boldsymbol{S}_{k \ell}=\frac{\sum_{i=1}^{n} \tilde{X}_{i k} \tilde{X}_{i \ell}}{n}-\frac{\sum_{i \neq j}^{n} \tilde{X}_{i k} \tilde{X}_{j \ell}}{n(n-1)}
$$

which is equal to (9) when $\pi_{k \ell}=\pi_{k}=1$ for all $k, \ell$. The following theorem shows the concentration of $\widehat{\mathbf{\Sigma}}^{I P W \mu}$.

Theorem 2. Assume the conditions of Lemma 1 hold except a mean zero condition, and further assume the sample size and dimension satisfy

$$
n / \log p>c_{2} \cdot \max \left\{\frac{1}{R\left(2 K / \sqrt{v_{\min }}\right)}, 1\right\},
$$

then

$$
\mathrm{P}\left[\left\|\widehat{\boldsymbol{\Sigma}}^{I P W \mu}-\boldsymbol{\Sigma}\right\|_{\max } \geq C_{2} \sqrt{\frac{\log p}{n}}\right] \leq d_{2} p^{-1},
$$

where $c_{2}>0, d_{2}>0$ are scalars. $C_{2}>0$ is a scalar quantity such that

$$
C_{2} \propto \frac{\max \left\{\sigma_{\max } K^{2} \sqrt{R(K)}, \mu_{\max } \sqrt{1+2 \sigma_{\max } e^{2} K^{2}}, \mu_{\max }^{2}\right\}}{\pi_{\min }}
$$

where $\mu_{\max }=\max _{k}\left|\mu_{k}\right|$.

A proof of Theorem 2 can be found in Section B.4 of Appendix, where we introduce a new version of Hanson-Wright inequality by extending the result of [38]. While the previous research deals with a quadratic form $X^{\mathrm{T}} A X$ of a sub-Gaussian vector $X$, we handle $X_{1}^{\mathrm{T}} A X_{2}$ of sub-Gaussian vectors $X_{1}$ and $X_{2}$ whose pair $\left(X_{1 i}, X_{2 i}\right)$ may be dependent with each other. We later notice that [50] considers missing indicators in the quadratic form $(X * \delta)^{\mathrm{T}} A(X * \delta)$. However, the result from [50] cannot be used to derive the rate in covariance estimation. It only enables one to calculate the convergence rate for diagonal components (i.e., marginal variances), since off-diagonal components are of form $\left(X_{1} * \delta_{1}\right)^{\mathrm{T}} A\left(X_{2} * \delta_{2}\right)$ for distinct $X_{1} \neq X_{2}$ and $\delta_{1} \neq \delta_{2}$ where $*$ is the element-wise product of two vectors.

As functions of missing probabilities, the sample size condition and convergence rate of Theorem 2 are not much different from those of Theorem 1 . This similarity is reasonable, because they both assume known missing probabilities. Further, the rate of convergence changes depending on the magnitude of mean parameters if the mean $\mu_{\max }$ is larger than the marginal variances. Supposedly, the mean parameter can be removed from the above formula for $C_{2}$ if missing values are imputed by the empirical mean of available data. However, we leave this as future work. 


\subsection{The case of unknown missing probability}

In real applications, the missing probability $\pi_{j k}$ is rarely known and needs to be estimated. Let $\hat{\pi}_{j k}$ be any estimate satisfying $\hat{\pi}_{j k}>0, \forall j, k$, with high probability. Then, the resulting IPW estimator is presented by

$$
\widehat{\mathbf{\Sigma}}^{I P W \pi}=\left(\left(\widehat{\boldsymbol{\Sigma}}^{I P W}\right)_{j k} \frac{\pi_{j k}}{\hat{\pi}_{j k}}, 1 \leq j, k \leq p\right),
$$

provided that the population mean is known for the sake of simplicity. When additional information on missingness is not available for estimating $\pi_{j k}$, it is natural to use the empirical proportions $\hat{\pi}_{j k}^{e m p}=n^{-1} \sum_{i=1}^{n} \delta_{i j} \delta_{i k}$ of observed samples since it is asymptotically unbiased for $\pi_{j k}$ (by the law of large numbers). We denote the empirical version $\widehat{\boldsymbol{\Sigma}}^{e m p}$ of the IPW estimator by

$$
\left(\widehat{\boldsymbol{\Sigma}}^{e m p}\right)_{j k}=\frac{\sum_{i=1}^{n} \delta_{i j} \delta_{i k} X_{i j} X_{i k}}{\sum_{i=1}^{n} \delta_{i j} \delta_{i k}}, \quad 1 \leq j, k \leq p,
$$

which corresponds to (10) with $\hat{\pi}_{j k}^{e m p}$ in place of $\hat{\pi}_{j k}$. One may realize the equivalence of the empirical estimate (11) to a pairwise complete analysis. Based on Lemma 8 that describes the concentration for the inverse probability of $\hat{\pi}_{j k}^{e m p}$, we can derive the concentration inequality of $\widehat{\boldsymbol{\Sigma}}^{e m p}$.

Theorem 3. Assume the conditions of Lemma 1 without knowing missing probabilities hold, and further assume the sample size and dimension satisfy

$$
n / \log p>c_{3} \cdot \max \left\{\frac{1}{R\left(2 K / \sqrt{v_{\min }}\right)}, \frac{1}{\pi_{\min }^{2}}\right\},
$$

then

$$
\mathrm{P}\left[\left\|\widehat{\boldsymbol{\Sigma}}^{e m p}-\boldsymbol{\Sigma}\right\|_{\max } \geq \frac{C_{3} \sigma_{\max } \max \left\{K^{2} \sqrt{R(K)}, 1\right\}}{\pi_{\min }^{2}} \sqrt{\frac{\log p}{n}}\right] \leq d_{3} p^{-1},
$$

where $c_{3}>0, d_{3}>0, C_{3}>0$ are scalars.

A proof of the theorem can be found in Section B.6 of Appendix.

As functions of missing probabilities, the sample size condition and the convergence rate change by the rate of $1 / \pi_{\min }^{2}$, meaning that they are more sensitive to missingness than in Theorem 1 . We believe such sensitiveness is the price paid for estimating unknown missing probabilities. We remark that this is observed via our simulation study (see Fig 7 and 8). Moreover, Theorem 3 has an implication that the convergence rate $\sqrt{\log p / n}$ in Theorem 1 is preserved, and thus the same statements in Theorem 2 hold true with $\widehat{\boldsymbol{\Sigma}}^{e m p}$. It should be pointed out that [23] use the estimator $\widehat{\boldsymbol{\Sigma}}^{e m p}$, but their theory is limited to the independent missing structure. Thus, Theorem 3 generalizes the theory for the empirical IPW estimator to the dependent structure. 


\section{Non-positive semi-definiteness of the plug-in estimator}

Despite its straightforward derivation and applicability to multivariate procedures in the presence of missing data, the IPW estimator has one critical issue from a practical point of view; non-positive semi-definiteness (non-PSDness). Note that this does not cause problems in the convergence rate, since the norm is element-wisely defined. It is well known that the element-wise product of two matrices may not preserve a nice property of the matrices. As addressed in high-dimensional covariance estimation (thresholding, banding, and tapering) $([3,37])$, the positive semi-definiteness is one of the typical examples to be broken down by the Hadamard product of a positive semi-definite (PSD) matrix and a general matrix. This is also the case for the IPW estimator, which makes it practically difficult to use the IPW estimator when using existing algorithms for estimating a precision matrix. For instance, we can plug the IPW estimator into the graphical lasso or the CLIME to estimate a sparse precision matrix $\boldsymbol{\Omega}=\left(\omega_{k \ell}, 1 \leq k, \ell \leq p\right)$, when missing data occur. However, the popularly used algorithms (glasso package or clime package in $\mathrm{R}$ ) require the plugged-in estimator to be positive semi-definite. In this section, we examine the graphical lasso algorithm from this point of view and also suggest possible solutions. A similar discussion about the CLIME can be found in Section C.1 of Appendix.

\section{Graphical lasso}

In what follows, we distinguish between a plug-in matrix (estimator) $\widehat{\mathbf{\Sigma}}^{\text {plug }}$ and an initial matrix (estimator) $\boldsymbol{\Sigma}^{(0)}$ (or $\boldsymbol{\Omega}^{(0)}$ ) that is used to initialize iterative steps.

The graphical lasso proposed by [13] aims to maximize the penalized likelihood function

$$
\max _{\boldsymbol{\Omega} \succeq 0}\left\{\log |\boldsymbol{\Omega}|-\operatorname{tr}\left(\boldsymbol{\Omega} \widehat{\boldsymbol{\Sigma}}^{\text {plug }}\right)-\lambda \sum_{k, \ell}\left|\omega_{k \ell}\right|\right\}
$$

for a penalty parameter $\lambda>0$. To solve (13), a coordinate descent algorithm described in Algorithm 1 is proposed by [13] and implemented in R package glasso. One can easily see that the optimization problem (13) is convex regardless of $\widehat{\boldsymbol{\Sigma}}^{\text {plug }}(\because$ the trace term is a linear function in $\boldsymbol{\Omega})$, but PSDness of $\widehat{\boldsymbol{\Sigma}}^{\text {plug }}$ is needed when the algorithm is initialized.

First, PDness of $\boldsymbol{\Sigma}^{(i-1)}$ is required in (14) to find a well-defined solution of the lasso problem. Since PD $\boldsymbol{\Sigma}^{(i-1)}$ guarantees the updated matrix $\boldsymbol{\Sigma}^{(i)}$ to be PD $([2])$, the PD initial $\boldsymbol{\Sigma}^{(0)}$ is necessary to make sure every step runs successfully. However, currently available $\mathrm{R}$ packages (e.g. glasso version 1.10 from [13] or huge version 1.3.2 from [49]) set $\boldsymbol{\Sigma}^{(0)} \leftarrow \widehat{\boldsymbol{\Sigma}}^{\text {plug }}+\lambda \mathbf{I}$ where $\lambda$ is the same parameter used in (13). As a consequence, unless $\lambda$ is bigger than the absolute value of the smallest (possibly negative) eigenvalue of $\widehat{\boldsymbol{\Sigma}}^{I P W}$, the coordinate descent algorithm would fail to converge. For this reason, we propose to use the 


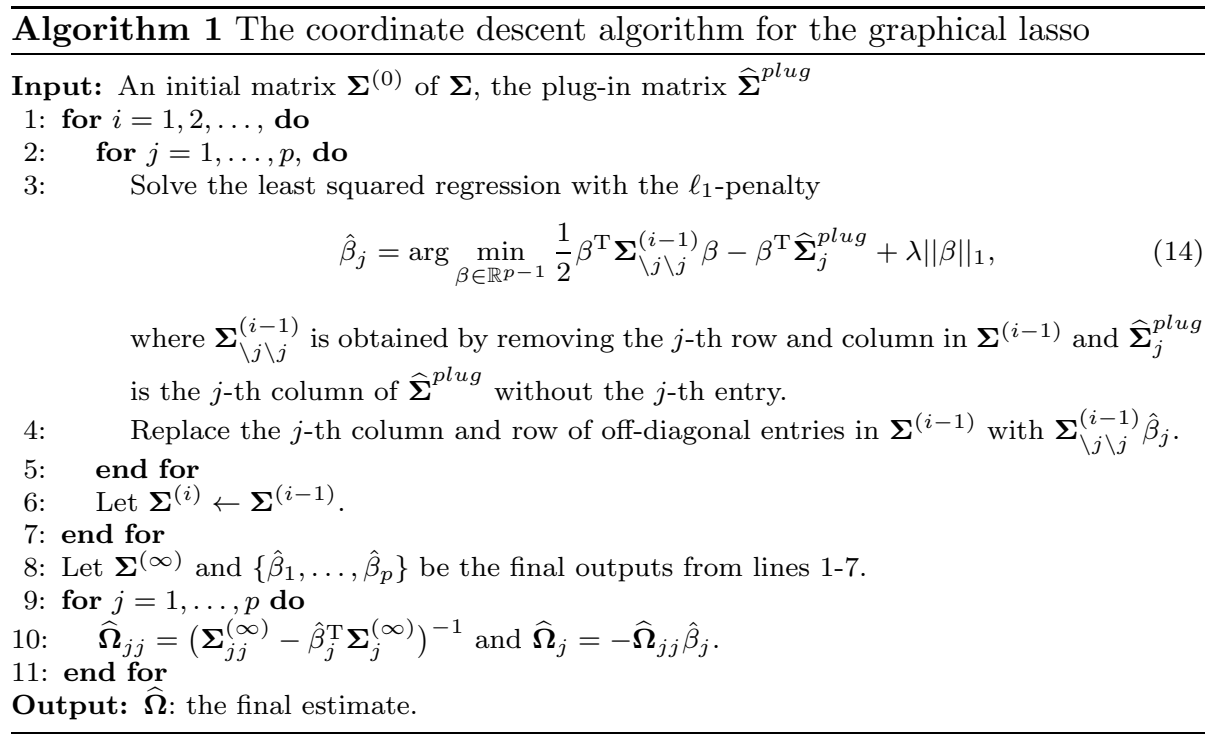

following inputs

$$
\widehat{\boldsymbol{\Sigma}}^{\text {plug }} \leftarrow \widehat{\boldsymbol{\Sigma}}^{I P W}, \quad \boldsymbol{\Sigma}^{(0)} \leftarrow \operatorname{diag}\left(\widehat{\boldsymbol{\Sigma}}^{I P W}+\lambda \mathbf{I}\right) .
$$

The above proposal for the initial matrix is made because diagonals of the solution $\boldsymbol{\Sigma}^{(\infty)}$ should satisfy $\boldsymbol{\Sigma}_{i i}^{(\infty)}=\widehat{\boldsymbol{\Sigma}}_{i i}^{\text {plug }}+\lambda, \forall i$, by the subgradient condition of (13), as noted in [13], and because diagonals of $\boldsymbol{\Sigma}^{(i)}$ do not change as iterations proceed. To use these proposed inputs, one should modify the off-the-shelf code (e.g. glasso function in glasso package) since it does not currently allow users to control $\boldsymbol{\Sigma}^{(0)}$ and $\widehat{\boldsymbol{\Sigma}}^{\text {plug }}$ individually.

Last but not least, it should be remarked that there is an algorithm developed to solve (13) by approximating the Hessian function (R package QUIC from [18]). This method does not suffer from the PSDness issue discussed here, which is verified through a numerical experiment given in Section C.2 of Appendix. However, solving the similar issue in the other multivariate procedures remains open.

\section{More general solution: matrix approximation}

Previously, we present the solutions that are specific to the precision matrix estimation problem, but we can circumvent the non-PSD issue for general statistical procedures. The idea is to approximate $\widehat{\boldsymbol{\Sigma}}^{\text {plug }}$ by the nearest PSD matrix, which can be achieved by

$$
\widehat{\boldsymbol{\Sigma}}^{\text {psd }}=\arg \min _{\boldsymbol{\Sigma} \succeq 0} d\left(\boldsymbol{\Sigma}, \widehat{\boldsymbol{\Sigma}}^{\text {plug }}\right)
$$

where $d$ measures the distance between two matrices. For instance, the Frobenius norm $([46,21])$ and the element-wise maximum norm $([27])$ are used pre- 
viously. Then, the nearest matrix $\widehat{\mathbf{\Sigma}}^{\text {psd }}$ would be put into the subsequent multivariate analyses (e.g. the graphical lasso) without modification in the current implementations. However, solving the problem (16) comes at the price of such convenience.

When the Frobenius norm is used, (16) amounts to a well-known projection onto the convex cone of PSD matrices. The solution denoted by $\widehat{\boldsymbol{\Sigma}}_{F}^{p s d}$ can be explicitly expressed by

$$
\widehat{\boldsymbol{\Sigma}}_{F}^{p s d}=\boldsymbol{V} \boldsymbol{W}_{+} \boldsymbol{V}^{\mathrm{T}}, \quad \boldsymbol{W}_{+}=\max (\boldsymbol{W}, \mathbf{0})
$$

where $\widehat{\boldsymbol{\Sigma}}^{\text {plug }}$ has the spectral decomposition $\boldsymbol{V} \boldsymbol{W} \boldsymbol{V}^{\mathrm{T}}$ and the maximum between two matrices operates element-wisely. The computational cost for this case is mostly from the eigenvalue decomposition, but the convergence rates derived for the IPW estimator in terms of the element-wise maximum norm (e.g. Theorem 1) are not guaranteed for $\widehat{\boldsymbol{\Sigma}}_{F}^{\text {psd }}$.

In contrast, when $d$ is the element-wise maximum norm ([27]), the convergence rate is preserved for the solution $\widehat{\boldsymbol{\Sigma}}_{M}^{p s d}$ since

$\left\|\widehat{\boldsymbol{\Sigma}}_{M}^{\text {psd }}-\boldsymbol{\Sigma}\right\|_{\max } \leq\left\|\widehat{\boldsymbol{\Sigma}}_{M}^{\text {psd }}-\widehat{\boldsymbol{\Sigma}}^{\text {plug }}\right\|_{\max }+\left\|\widehat{\boldsymbol{\Sigma}}^{\text {plug }}-\boldsymbol{\Sigma}\right\|_{\max } \leq 2\left\|\widehat{\boldsymbol{\Sigma}}^{\text {plug }}-\boldsymbol{\Sigma}\right\|_{\max }$ where the first inequality uses the triangular inequality and the second is from the definition of $\widehat{\boldsymbol{\Sigma}}_{M}^{p s d}$. The algorithm to solve (16) with the element-wise maximum norm is first proposed by [47] and used in the robust covariance estimation context $([27,16])$. We note, however, by experience that the approximation based on $\|\cdot\|_{\max }$ is computationally heavy so that it often dominates the computation time of multivariate procedures (e.g. the graphical lasso and the CLIME). On the other hands, $[15,10]$ use the alternating direction method of multipliers (ADMM) to solve (16) with the element-wise maximum norm.

\section{Numerical study}

In this section, we perform a number of simulations for estimating a covariance/precision matrix with partially observed data. First, in Section 5.2, we experimentally check the convergence rate of the IPW estimator given in our theorems. In Section 5.3, we conduct a comparison study between several imputation methods and the IPW method. Performance of the estimates is also measured and compared according to simulation parameters, and the related results can be found in Section D.2 of Appendix.

\subsection{Setting}

Data generation

We generate Gaussian random vectors $X_{i}, i=1, \ldots, n$, in $\mathbb{R}^{p}$ with mean vector 0 and precision matrix $\boldsymbol{\Omega}=\left(\omega_{i j}, 1 \leq i, j \leq p\right)$ under different pairs of $n=$ $50,100,200$ and $p$ satisfying $r(=p / n)=0.2,1,2$. We consider three types of 
precision matrix as follows, which have been used in the previous literature ([5, 28]): chain, star, random graphs. Two structures (independent, dependent) are under consideration to impose missingness on data where the missing proportion is set to $0 \%, 15 \%, 30 \%$. More precise definitions of true precision matrices and missing structures are given in Section D.1 of Appendix.

\section{Estimators}

We compare two types of plug-in estimator: $\widehat{\boldsymbol{\Sigma}}^{I P W}$, an oracle type estimator labeled by "orc" and $\widehat{\boldsymbol{\Sigma}}^{e m p}$, an empirical type estimator labeled by "emp". A closed form of the weight $\pi_{k \ell}$ is accessible according to each missing structure, so the oracle IPW estimator is explicitly computable. It is noteworthy that the estimator $\widehat{\boldsymbol{\Sigma}}^{e m p}$ is used in [23], but their theoretical analysis is limited to the independent missing structure.

We exploit QUIC algorithm proposed by [18] to solve the graphical lasso (13). The grid of a tuning parameter $\lambda \in \Lambda$ is defined adaptively to the plug-in matrix $\widehat{\Sigma}^{\text {plug }}$.

\subsection{The rate of convergence}

We verify our theoretical results (Theorem 1 and 3 ) by computing the elementwise maximum deviation $\| \widehat{\boldsymbol{\Sigma}}^{\text {plug }}-\left.\boldsymbol{\Sigma}\right|_{\max }$. We fix $p=100$ and vary the sample size in $20 \leq n \leq 10000$. We repeat each scenario 20 times and plot the logtransformed empirical distance against $\log (n / p)$. Different plug-in estimators ("orc", "emp") and precision matrices (chain, star, random) are under consideration.

Figure 1 shows that each graph connecting the averaged distances nearly forms a straight line. The results in the column "orc" confirm the rate of convergence in Theorem 1, while those in the column "emp" confirms that in Theorem 3.

\subsection{Comparison with imputation methods}

In the missing data context, unobserved data is often substituted by some function of observed values. One very intuitive way to do it is the imputation method. Once the pseudo complete data is produced, we perform a usual statistical analysis. In this experiment, we compare different (single) imputation approaches with the IPW estimator for the precision matrix estimation.

Imputation methods we use are "median" (a median of available data for each variable), "pmm" (predictive mean matching from R package Hmisc ([20])), "knn" (an average of k-nearest neighbors from R package impute ([17])), "cart", "rf", and "norm" (regression-based methods from R package mice ([43])). We use the default parameter setting for each $\mathrm{R}$ function. More details of each method can be found in each reference. 


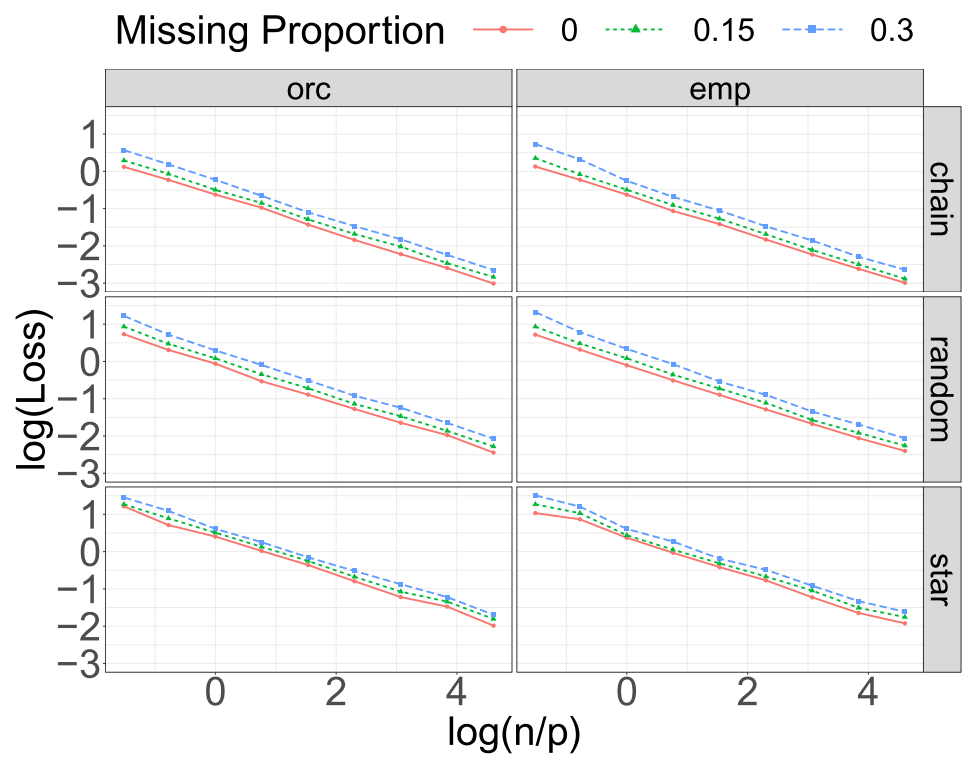

FIG 1. Convergence rate of the plug-in matrix ( "orc" $=\widehat{\boldsymbol{\Sigma}}^{I P W}$, "emp" $=\widehat{\boldsymbol{\Sigma}}^{e m p}$ ) against $\log (n / p)$. Loss is computed by the element-wise maximum norm between the plug-in matrix and the true covariance matrix. The dependent missing structure and $p=100$ are assumed. Each dot (or mark) is an average loss from 20 repetitions.

By fixing $n=100$ and $r=1,2$, we randomly generate 100 data sets based on different precision matrices. Missing observations are produced under the independent structure. Once missing observations are filled by a single imputation method, then we compute the sample covariance matrix with the imputed complete data and carry out the precision matrix estimation using the QUIC algorithm. We compare the competing methods based on support recovery of the estimated precision matrix. Figure 2 shows the pAUC values, where the IPW method using the empirical estimator ("emp") achieves the largest pAUC compared to the imputation approaches. This is more distinct when the dimension is larger than the sample size (i.e., $r=2$ ). The results demonstrate that the IPW method is not only theoretically solid, but also practically useful. Admittedly, we have not thoroughly examined more diverse and complex imputation methods that may produce better performance, which calls for extensive numerical studies in the future.

\subsection{Unknown mean}

In this last experiment, a mean vector is no longer assumed to be zero and thus one needs to consider the corresponding estimator $\widehat{\mathbf{\Sigma}}^{I P W \mu}$ given in (9). The goal of this simulation is to check how the estimation error of (9) changes according to the mean vector. We consider two types of structures in it: "full" 


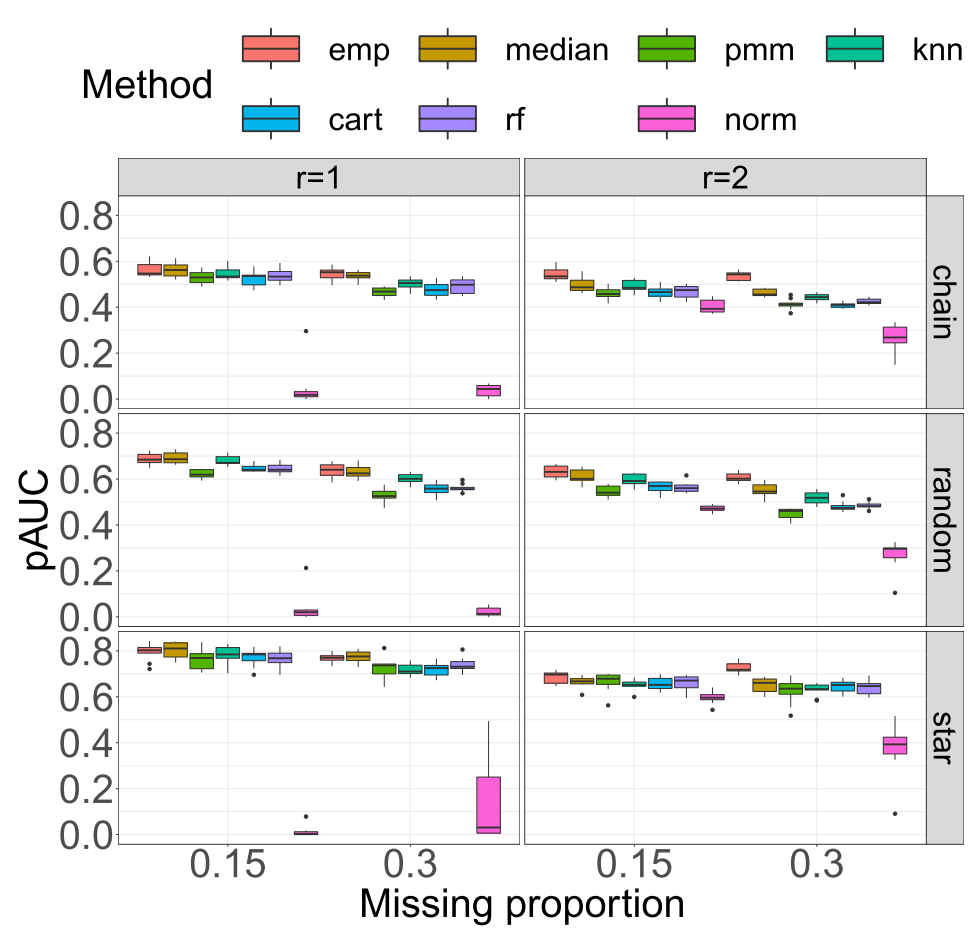

FIG 2. Comparison of the pAUC values for different approaches to handle missingness in estimating a sparse precision matrix. Here, $r=1,2, n=100$, and the independent missing structure are assumed. The empirical IPW estimator is plugged-in. We randomly generate 10 data sets.

$\left(\mu=k(1, \ldots, 1)^{\mathrm{T}}\right)$ and "sparse" $\left(\mu=k(1, \ldots, 1,0, \ldots, 0)^{\mathrm{T}}\right)$. The sparse vector has zeros in the last half of its components. The magnitude $k>0$ is set by the size condition $\|\mu\|_{2}=1,2,4,8$. In Figure 3 , the case when size of mean is 0 indicates when the mean vector is known, so it is included as a control group. The proportion of missing data varies over $15,30 \%$.

We measure the estimation error by the element-wise maximum norm $\| \widehat{\mathbf{\Sigma}}^{I P W \mu}$ $\boldsymbol{\Sigma}||_{\max }$ to verify the bound in Theorem 2 . The estimation error does not increase by replacing the true means with their empirical estimates if the size of the true mean $\left(=\max \left\{\mu_{\max }, \mu_{\max }^{2}\right\}\right)$ is not large and less than some cut-off value $\left(=\sigma_{\max }\right)$. However, if the size of the true mean is large, the estimation error starts to increase. A larger estimation error in the sparse structure of mean compared to the full can be also explained. Given the same 2-norm $\|\mu\|_{2}$, the sparse vector has larger $\mu_{\max }$ than the full vector due to the sparsity, which results in more deviation.

The above observations are also valid in terms of the estimation of a precision matrix where $\widehat{\mathbf{\Sigma}}^{I P W \mu}$ is plugged-in. The related results are given in Appendix D. 2 


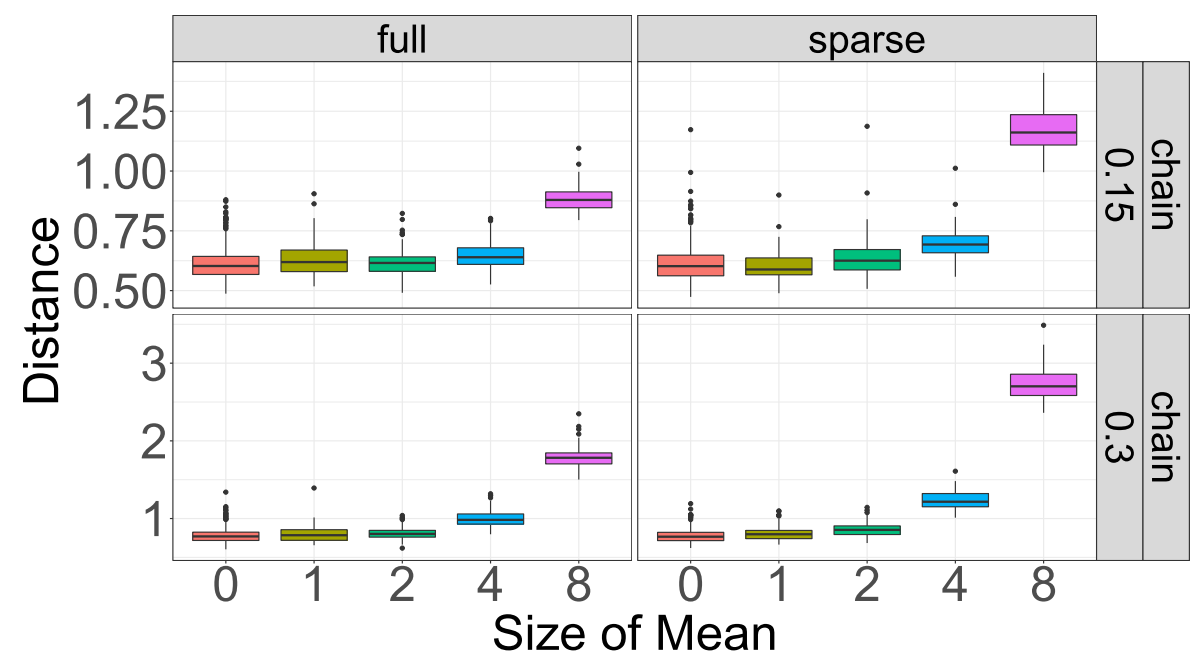

FIG 3. Element-wise maximum norm $\left\|\widehat{\boldsymbol{\Sigma}}^{I P W \mu}-\boldsymbol{\Sigma}\right\|_{\max }$ according to a magnitude of (unknown) mean. Here, $n=100, r=1$, the chain structure in $\Omega$, and the dependent missing structure are assumed. We randomly generate 100 data sets.

\section{Application to real data}

We examine the estimation performance of the IPW estimator through a real data application. We use the riboflavin data available from the $\mathrm{R}$ package hdi, where 4088 gene expressions are observed across 71 samples. Since the ground truth precision matrix is not known, we construct it by solving the graphical lasso (13) at a fixed $\lambda$ with a complete data. We impose missing values in a similar manner described in Section 5. Throughout this analysis, it is confirmed again that having more missing values yields worse estimation. Also, it is possible to see that the denser model that has a precision matrix with more non-zero elements is more difficult to achieve satisfactory accuracy in estimation and graph recovery. More details and results can be found in Section E of Appendix.

\section{Discussion}

This paper considers a theoretical establishment of the IPW estimator with missing observations. Contrary to the previous literature, this is achieved under dependency among missingness, meaning that missing indicators are not necessarily independent across variables. The rate of convergence of the IPW estimator is derived based on the element-wise maximum norm, which is (asymptotically) in the same order of the rate claimed in the past works. Our analysis can be applied to an estimation of a sparse precision matrix. Due to the metatheorem, the favorable properties (consistency, support recovery) of the final estimator are preserved in the missing data context. 
The plug-in estimators (e.g. the sample covariance matrix and the IPW estimator) and their concentration are often not of primary interest, but the ultimate goal lies in applying them to downstream procedures (e.g. Hotelling's $\mathrm{T}^{2}$, a portfolio optimization, etc). In the portfolio optimization, [12] show that the risk inequality is bounded by the error of the plug-in estimator $\widehat{\boldsymbol{\Sigma}}^{\text {plug }}$;

$$
\left|w^{\mathrm{T}} \widehat{\boldsymbol{\Sigma}}^{\text {plug }} w-w^{\mathrm{T}} \boldsymbol{\Sigma} w\right| \leq\left\|\widehat{\boldsymbol{\Sigma}}^{\text {plug }}-\boldsymbol{\Sigma}\right\|_{\max } .
$$

Here, $w$ and $\boldsymbol{\Sigma}$ are true (or optimal) parameters. However, it is still elusive how the rate $\|\hat{w}-w\|_{V}$ for the optimal solution $\hat{w}$ that minimizes the risk $t \mapsto t^{\mathrm{T}} \widehat{\boldsymbol{\Sigma}}^{\text {plug }} t$ is linked to the rate $\left\|\widehat{\boldsymbol{\Sigma}}^{\text {plug }}-\boldsymbol{\Sigma}\right\|_{M}$ of the plug-in estimator. $\|\cdot\|_{V}$ and $\|\cdot\|_{M}$ are some norms of a vector and a matrix, respectively. This line of research could be interesting for future work and in urgent need, not to mention its extension to the missing data context.

The underlying assumptions on the missing mechanism (i.e., MCAR) and the missing structure (i.e., identical dependency across samples) are essentially not verifiable, but it is natural to think of extending our results to the cases beyond such patterns. For example, missing at random (MAR) mechanism assumes that missingness occurs independently of unobserved random variables given observed variables. Then, it is easy to show the corresponding IPW estimator is still unbiased. However, it is not straightforward to follow the analyses given in this paper to this case. It would be interesting to identify suitable assumptions that are less stronger than MCAR, but still guarantee the missing probabilities to be free from $X_{i, o b s}$.

\section{Appendix A: Auxiliary lemmas}

The first supporting lemma tells a tail bound of a variable with a cumulant generating function dominated by a quadratic function.

Lemma 2 (Theorem 3.2 and Lemma 2.4 in [39]). Let a random variable $\xi_{j}$ with $\mathbb{E} \xi_{j}=0, \operatorname{Var}\left(\xi_{j}\right)=\sigma_{j}^{2}$ satisfy the following; there exist positive constants $A, C, c_{1}, c_{2}, \ldots$, such that

$$
\left|\log \mathbb{E} \exp \left\{\lambda \xi_{j}\right\}\right| \leq c_{j}^{2} \lambda^{2}, \quad|\lambda|<A, \quad \forall j,
$$

and

$$
\varlimsup_{n \rightarrow \infty} \sum_{j=1}^{n} c_{j}^{2} / \sum_{j=1}^{n} \sigma_{j}^{2} \leq C .
$$

Then, we have for $\xi=\sum_{j=1}^{n} \xi_{j} / \sqrt{\sum_{j=1}^{n} \sigma_{j}^{2}}$,

$$
\mathrm{P}[ \pm \xi \geq x] \leq \exp \left(-x^{2} / 8 C\right), \quad 0 \leq x \leq 2 A C \sqrt{\sum_{j=1}^{n} \sigma_{j}^{2}} .
$$


Furthermore, if $\xi_{i}$ 's are identically distributed and satisfying the conditions above, then the variance term $\sigma_{j}^{2}$ does not appear in the concentration inequality:

$$
\mathrm{P}\left[ \pm \sum_{j=1}^{n} \xi_{j} \geq x\right] \leq \exp \left\{-\frac{x^{2}}{8 n c_{1}^{2}}\right\}, \quad 0 \leq x \leq 2 A n c_{1}^{2} .
$$

The following auxiliary results for a sub-Gaussian variable $X$ facilitate one to check the condition (17) in Lemma 4.

Lemma 3. Assume that $X$ is a random variables satisfying Assumption 1 for some $K>0$. Then, it holds

(a) for $|t| \leq(2 e K)^{-1}$,

$$
\mathbb{E} \exp (t X) \leq \exp \left\{\left(1 / 2+K^{2} e^{2}\right) t^{2}\right\},
$$

(b) and for $|t|<1 /(2 \kappa)$,

$$
\mathbb{E}\left[\exp \left\{t\left(X^{2}-1\right)\right\}\right] \leq \exp \left(c_{0} t^{2}\right),
$$

where $\kappa=4 e K^{2}$ and $c_{0}=2 \kappa^{2}\{\exp (1 / \kappa)-1 / 2-1 / \kappa\}$.

Proof. We first prove (a). For $t \in \mathbb{R}$, observe that

$$
\begin{aligned}
\mathbb{E} \exp (t X) & =1+\frac{t^{2}}{2}+\sum_{r \geq 3} \frac{\mathbb{E} X^{r} t^{r}}{r !} \\
& \leq 1+\frac{t^{2}}{2}+\sum_{r \geq 3} \frac{\mathbb{E}|X|^{r} t^{r}}{r !} \\
& \leq 1+\frac{t^{2}}{2}+\sum_{r \geq 3} \frac{K^{r} r^{r}|t|^{r}}{r !} \\
& \leq 1+\frac{t^{2}}{2}+\sum_{r \geq 3} K^{r} e^{r}|t|^{r} \quad\left(\because(r / e)^{r} \leq r !\right) \\
& \leq 1+\frac{t^{2}}{2}+\frac{(K e|t|)^{3}}{1-K e|t|}, \quad \text { if }|t| \leq(K e)^{-1} .
\end{aligned}
$$

Then, it holds for any $0<t_{0}<(K e)^{-1}$ that for all $|t|<t_{0}$,

$$
\mathbb{E} \exp (t X) \leq 1+|t|^{2}\left(1 / 2+K^{2} e^{2}\right) \leq \exp \left\{|t|^{2}\left(1 / 2+K^{2} e^{2}\right)\right\},
$$

which concludes the proof of (a).

Next, we prove (b). Using the Minkowski inequality, we have

$$
\left(\mathbb{E}\left|X^{2}-1\right|^{r}\right)^{1 / r} \leq\left(\mathbb{E}|X|^{2 r}\right)^{1 / r}+1 \leq 2 r K^{2}+1,
$$

which thus gives the upper bound of moments of $X^{2}-1$,

$$
\mathbb{E}\left|X^{2}-1\right|^{r} \leq\left(2 r K^{2}+1\right)^{r} \leq 2^{r-1}\left(2^{r} r^{r} K^{2 r}+1\right) .
$$


Therefore,

$$
\begin{aligned}
\mathbb{E}\left[\exp \left\{t\left(X^{2}-1\right)\right\}\right] & =1+\sum_{r \geq 2} \frac{t^{r} \mathbb{E}\left(X^{2}-1\right)^{r}}{r !} \\
& \leq 1+\sum_{r \geq 2} \frac{|t|^{r} 2^{r-1}\left(2^{r} r^{r} K^{2 r}+1\right)}{r !} \\
& \leq 1+\frac{1}{2} \sum_{r \geq 2}\left\{\frac{\left(4|t| r K^{2}\right)^{r}}{r !}+\frac{(2|t|)^{r}}{r !}\right\} \\
& \leq 1+\frac{1}{2} \sum_{r \geq 2}\left\{\left(4|t| e K^{2}\right)^{r}+\frac{(2|t|)^{r}}{r !}\right\} \\
& =1+\frac{|t|^{2}}{2} \sum_{r \geq 2}\left\{\left(4 e K^{2}\right)^{2}\left(4|t| e K^{2}\right)^{r-2}+\frac{4(2|t|)^{r-2}}{r !}\right\}
\end{aligned}
$$

where the last inequality is derived from $(n / e)^{n} \leq n$ ! for $n \geq 1$. Then, it holds for any $0<t_{0}<1 /\left(4 e K^{2}\right)$ that for all $|t|<t_{0}$,

$$
\mathbb{E}\left[\exp \left\{t\left(X^{2}-1\right)\right\}\right] \leq 1+c t^{2} \leq \exp \left(c t^{2}\right)
$$

where $c$ is a function of $t_{0}$ defined by

$$
c=c\left(t_{0}\right)=\frac{1}{2} \sum_{r \geq 0}\left\{\left(4 e K^{2}\right)^{2}\left(4 t_{0} e K^{2}\right)^{r}+\frac{4\left(2 t_{0}\right)^{r}}{(r+2) !}\right\} .
$$

Calculus of infinite series at the choice of $t_{0}=1 /\left(8 e K^{2}\right)$ gives

$$
c\left(t_{0}\right)=\frac{\exp \left(2 t_{0}\right)-1 / 2-2 t_{0}}{2 t_{0}^{2}},
$$

which concludes the proof of (b).

Lemma 4. Assume that $X$ is a random variables satisfying Assumption 1 for some $K>0$. Then, the i.i.d copies $X_{1}, \ldots, X_{n}$ of $X$ satisfy,

(a) for $0 \leq x \leq e K+(2 e K)^{-1}$,

$$
\mathrm{P}\left[\left|\sum_{j=1}^{n} X_{j}\right| \geq n x\right] \leq 2 \exp \left\{-\frac{n x^{2}}{8\left(1 / 2+K^{2} e^{2}\right)}\right\},
$$

(b) and for $0 \leq x \leq 4 e K^{2} R(K)$,

$$
\mathrm{P}\left[\left|\sum_{j=1}^{n}\left(X_{j}^{2}-1\right)\right| \geq n x\right] \leq 2 \exp \left\{-\frac{n x^{2}}{16\left(4 e K^{2}\right)^{2} R(K)}\right\},
$$

where $R(t)=\exp \left\{1 /\left(4 e t^{2}\right)\right\}-1 / 2-1 /\left(4 e t^{2}\right), t>0$.

Proof. The proofs of (a) and (b) directly come from applications of Lemma 2 and 3 . 


\section{Appendix B: Proofs}

\section{B.1. Proof of Lemma 1}

Proof of Lemma 1. Assume $k$ and $\ell$ are distinct. We start by decoupling the product of two sub-Gaussian variables $Y_{i k} Y_{i \ell} / \pi_{k \ell}$ using an identity $x y=\{(x+$ $\left.y)^{2}-(x-y)^{2}\right\} / 4$ so that we have for $t \geq 0$,

$$
\left\{\left|\sum_{i=1}^{n}\left(\frac{Y_{i k} Y_{i \ell}}{\pi_{k \ell}}-\sigma_{k \ell}\right)\right| \geq n t\right\} \subset A \cup B,
$$

where $Y_{i k}^{*}=Y_{i k} / \sqrt{\sigma_{k k}}$ and

$$
\begin{aligned}
& A=\left\{\left|\sum_{i=1}^{n}\left\{\left(Y_{i k}^{*}+Y_{i \ell}^{*}\right)^{2}-\mathbb{E}\left(Y_{i k}^{*}+Y_{i \ell}^{*}\right)^{2}\right\}\right| \geq \frac{2 n \pi_{k \ell} t}{\sqrt{\sigma_{k k} \sigma_{\ell \ell}}}\right\}, \\
& B=\left\{\left|\sum_{i=1}^{n}\left\{\left(Y_{i k}^{*}-Y_{i \ell}^{*}\right)^{2}-\mathbb{E}\left(Y_{i k}^{*}-Y_{i \ell}^{*}\right)^{2}\right\}\right| \geq \frac{2 n \pi_{k \ell} t}{\sqrt{\sigma_{k k} \sigma_{\ell \ell}}}\right\} .
\end{aligned}
$$

Let $v_{k \ell}=\mathbb{E}\left|Y_{i k}^{*}+Y_{i \ell}^{*}\right|^{2}=\pi_{k}+\pi_{\ell}+2 \pi_{k \ell} \rho_{k \ell}$. To apply Lemma 4 in Supplementary Material, we first show $Y_{i k}^{*}+Y_{i \ell}^{*}$ is a sub-Gaussian variable satisfying the conditions of the lemma.

Fact. For $i=1, \ldots, n$ and $1 \leq k \neq \ell \leq p$, we have

$$
\sup _{r \geq 1} \frac{\left\{\mathbb{E}\left|Y_{i k}+Y_{i \ell}\right|^{r}\right\}^{1 / r}}{\sqrt{r v_{k \ell}}} \leq 2 K / \sqrt{v_{k \ell}} .
$$

Proof. To obtain an uniform bound on higher moments, we observe that

$$
\begin{aligned}
\frac{\left\{\mathbb{E}\left|Y_{i k}^{*}+Y_{i \ell}^{*}\right|^{r}\right\}^{1 / r}}{\sqrt{r}} & \leq \frac{2^{1-1 / r}\left\{\mathbb{E}\left|Y_{i k}^{*}\right|^{r}+\mathbb{E}\left|Y_{i \ell}^{*}\right|^{r}\right\}^{1 / r}}{\sqrt{r}} \\
& =\frac{2^{1-1 / r}\left\{\pi_{k} \mathbb{E}\left|X_{i k} / \sqrt{\sigma_{k k}}\right|^{r}+\pi_{\ell} \mathbb{E}\left|X_{i \ell} / \sqrt{\sigma_{\ell \ell}}\right|^{r}\right\}^{1 / r}}{\sqrt{r}} \\
& \leq \frac{2^{1-1 / r}\left\{\pi_{k}(\sqrt{r} K)^{r}+\pi_{\ell}(\sqrt{r} K)^{r}\right\}^{1 / r}}{\sqrt{r}} \\
& \leq 2 K\left(\frac{\pi_{k}+\pi_{\ell}}{2}\right)^{1 / r}
\end{aligned}
$$

where the first inequality holds due to convexity of $x \mapsto|x|^{r}(r \geq 1)$ and the third inequality uses the moment condition of the sub-Gaussian variable $X_{i k} / \sqrt{\sigma_{k k}}$. We note that $\left(\frac{\pi_{k}+\pi_{\ell}}{2}\right)^{1 / r} \leq 1$ for all $r \geq 1$ since $0 \leq\left(\pi_{k}+\pi_{\ell}\right) / 2 \leq 1$. which concludes the proof. 
By applying Lemma 4 (b), we have for some numerical constants $c, C>0$,

$\mathrm{P}\left[\left|\sum_{i=1}^{n}\left\{\left(Y_{i k}^{*}+Y_{i \ell}^{*}\right)^{2}-v_{k \ell}\right\}\right| \geq \frac{2 n \pi_{k \ell} t}{\sqrt{\sigma_{k k} \sigma_{\ell \ell}}}\right] \leq 2 \exp \left\{-\frac{C n \pi_{k \ell}^{2} t^{2}}{K^{4} \sigma_{k k} \sigma_{\ell \ell} R\left(2 K / \sqrt{v_{k \ell}}\right)}\right\}$,

for $0 \leq t \leq \frac{c\left(\sigma_{k k} \sigma_{\ell \ell}\right)^{1 / 2} K^{2} R\left(2 K / \sqrt{v_{k \ell}}\right)}{\pi_{k \ell}}$. Hence, replacing $t$ by

$$
\tilde{t} \equiv \frac{\left(\sigma_{k k} \sigma_{\ell \ell}\right)^{1 / 2} K^{2} R\left(2 K / \sqrt{v_{k \ell}}\right)^{1 / 2}}{C^{1 / 2} \pi_{k \ell}} t, \quad t>0,
$$

in the above inequality, we get

$$
\begin{aligned}
\mathrm{P}\left[\left|\sum_{i=1}^{n}\left\{\left(Y_{i k}+Y_{i \ell}\right)^{2}-\mathbb{E}\left(Y_{i k}+Y_{i \ell}\right)^{2}\right\}\right| \geq 2 n \pi_{k \ell} \tilde{t}\right] \\
\leq 2 \exp \left\{-n t^{2}\right\}, \quad 0 \leq t \leq \tilde{c} \sqrt{R\left(2 K / \sqrt{v_{k \ell}}\right)},
\end{aligned}
$$

for some numerical constant $\tilde{c}>0$. Note that

$$
R\left(\frac{2 K}{\sqrt{\pi_{k}+\pi_{\ell}-2 \pi_{k \ell}\left|\rho_{k \ell}\right|}}\right) \leq R\left(\frac{2 K}{\sqrt{v_{k \ell}}}\right) \leq R(K),
$$

and using this bounds, we now have with probability at most $2 \exp \left\{-n t^{2}\right\}$

$$
\left|\sum_{i=1}^{n}\left\{\left(Y_{i k}+Y_{i \ell}\right)^{2}-\mathbb{E}\left(Y_{i k}+Y_{i \ell}\right)^{2}\right\}\right| \geq 2 n \pi_{k \ell} \frac{\left(\sigma_{k k} \sigma_{\ell \ell}\right)^{1 / 2} K^{2} R(K)^{1 / 2}}{C^{1 / 2} \pi_{k \ell}} t,
$$

for $0 \leq t \leq \tilde{c} \sqrt{R\left(\frac{2 K}{\sqrt{\pi_{k}+\pi_{\ell}-2 \pi_{k \ell}\left|\rho_{k \ell}\right|}}\right)}$. The similar statement holds with $Y_{i k}-Y_{i \ell}^{*}$. Therefore, combining these results with (18) yield

$$
\mathrm{P}\left[n^{-1}\left|\sum_{i=1}^{n}\left(\frac{Y_{i k} Y_{i \ell}}{\pi_{k \ell}}-\sigma_{k \ell}\right)\right| \geq \frac{\left(\sigma_{k k} \sigma_{\ell \ell}\right)^{1 / 2} K^{2} R(K)^{1 / 2}}{C^{1 / 2} \pi_{k \ell}} t\right] \leq 4 \exp \left\{-n t^{2}\right\},
$$

for $0 \leq t \leq \tilde{c} \sqrt{R\left(\frac{2 K}{\sqrt{\pi_{k}+\pi_{\ell}-2 \pi_{k \ell}\left|\rho_{k \ell}\right|}}\right)}$, which completes the proof for the case of $k \neq \ell$.

The concentration inequality for diagonal entries (i.e., $k=\ell$ ) of the IPW estimate is similarly derived. One can easily check

$$
\sup _{r \geq 1} \frac{\left\{\mathbb{E}\left|Y_{i k}\right|^{r}\right\}^{1 / r}}{\sqrt{r \pi_{k} \sigma_{k k}}} \leq K / \sqrt{\pi_{k}}
$$

Then, due to Lemma 4 (b), we get

$$
\mathrm{P}\left[n^{-1}\left|\sum_{i=1}^{n}\left(\frac{Y_{i k}^{2}}{\pi_{k}}-\sigma_{k k}\right)\right| \geq \frac{\tilde{C} \sigma_{k k} K^{2} R(K)^{1 / 2}}{\pi_{k}} t\right] \leq 2 \exp \left\{-n t^{2}\right\},
$$

for $0 \leq t \leq \sqrt{R\left(K / \sqrt{\pi_{k}}\right)}$. This concludes the whole proof. 


\section{B.2. Proof of Theorem 1}

Proof. From Lemma 1, it holds that for $1 \leq k, \ell \leq p$,

$$
\mathrm{P}\left[n^{-1}\left|\sum_{i=1}^{n}\left(\frac{Y_{i k} Y_{i \ell}}{\pi_{k \ell}}-\sigma_{k \ell}\right)\right| \geq \frac{C \sigma_{\max } K^{2} R(K)^{1 / 2}}{\pi_{\min }} t\right] \leq 4 \exp \left(-n t^{2}\right)
$$

if $t \geq 0$, since $R$ is monotonically decreasing,

$$
\begin{cases}t^{2} \leq c R\left(2 K / \sqrt{v_{\text {min }}}\right), & \text { if } k \neq \ell, \\ t^{2} \leq c R\left(K / \sqrt{\pi_{\text {min }, d}}\right) & \text { if } k=\ell,\end{cases}
$$

where $\pi_{m i n, d}=\min _{k} \pi_{k}$. Then, by using an union bound argument, we get

$$
\mathrm{P}\left[\max _{k, \ell}\left|\frac{1}{n} \sum_{i=1}^{n}\left(\frac{Y_{i k} Y_{i \ell}}{\pi_{k \ell}}-\sigma_{k \ell}\right)\right| \geq \frac{C \sigma_{\max } K^{2} \sqrt{R(K)} t}{\pi_{\min }}\right] \leq 4 p^{2} \exp \left(-n t^{2}\right) .
$$

for $t^{2} / c \leq R\left(K / \sqrt{\left(v_{\min } / 4\right) \wedge \pi_{\min , d}}\right)=R\left(2 K / \sqrt{\left.v_{\min }\right)}\right)$. Note that $v_{\text {min }} / 4 \leq$ $\pi_{\min , d}$.

Then, by plugging-in $t \leftarrow \alpha \sqrt{\log p / n}(\alpha>0)$, we get the convergence rate of the maximum norm of the IPW estimate,

$$
\mathrm{P}\left[\max _{k, \ell}\left|\left(\widehat{\boldsymbol{\Sigma}}^{I P W}\right)_{k \ell}-\sigma_{k \ell}\right| \geq \frac{C \sigma_{\max } K^{2} \alpha}{\pi_{\min }} \sqrt{\frac{R(K) \log p}{n}}\right] \leq 4 p^{2-\alpha^{2}},
$$

if $0 \leq \alpha^{2} \leq c R\left(2 K / \sqrt{v_{\min }}\right) n / \log p$. Suppose $n, p$ satisfy

$$
n / \log p>\frac{9}{c^{2} R\left(2 K / \sqrt{v_{\min }}\right)}
$$

so that we can choose $\alpha^{2}=3$. This concludes the proof.

\section{B.3. Proof of Corollary 2}

Proof. We summarize theorems/lemmas from the original works that bridge the rate of the plug-in estimator with those of the final precision matrix. If $\delta=$ $\sqrt{\log p / n}$ in each theorem, then the rates of the precision matrix are optimal and guarantee both estimation consistency in different norms and support recovery $\left(\because\|\cdot\|_{\max }\right)$. As usual, $\widehat{\boldsymbol{\Sigma}}^{\text {plug }}$ denotes the plug-in estimator.

\section{Graphical lasso}

Suppose $S \subset[p] \times[p]$ is an union of a true edge set and diagonal elements. Define $\boldsymbol{\Gamma}=\boldsymbol{\Omega}^{-1} \otimes \boldsymbol{\Omega}^{-1}$,

$$
\Gamma_{S S}=\left(\boldsymbol{\Omega}^{-1} \otimes \boldsymbol{\Omega}^{-1}\right)_{S S}=\boldsymbol{\Omega}_{S}^{-1} \otimes \boldsymbol{\Omega}_{S}^{-1},
$$


and similarly $\boldsymbol{\Gamma}_{e S}=\left(\boldsymbol{\Omega}^{-1} \otimes \boldsymbol{\Omega}^{-1}\right)_{e S}, e \in S^{c}$. Also, denote $\kappa_{\boldsymbol{\Sigma}}=\|\boldsymbol{\Sigma}\|_{\infty}$ and $\kappa_{\boldsymbol{\Gamma}}=\left\|\left(\boldsymbol{\Gamma}_{S S}\right)^{-1}\right\|_{\infty}$. $d$ is the maximum degree of the graph defined by $d=$ $\max _{i} \sum_{j} \mathrm{I}\left(\left|\omega_{i j}\right| \neq 0\right)$ and $s$ is the number of true edges.

Theorem (Lemmas 4, 5, 6, [35]). Assume the irrepresentability condition holds with degree of $\alpha \in(0,1]$

$$
\max _{e \in S^{c}}\left\|\boldsymbol{\Gamma}_{e S} \boldsymbol{\Gamma}_{S S}^{-1}\right\|_{1} \leq 1-\alpha .
$$

If $\left\|\widehat{\boldsymbol{\Sigma}}^{\text {plug }}-\boldsymbol{\Sigma}\right\|_{\max } \leq \delta=\delta_{n, p}$ and $n$ satisfies

$$
\delta_{n, p} \leq\left[6 d\left(1+8 \alpha^{-1}\right) \max \left\{\kappa_{\Gamma^{*}} \kappa_{\boldsymbol{\Sigma}^{*}}, \kappa_{\boldsymbol{\Gamma}^{*}}^{2} \kappa_{\boldsymbol{\Sigma}^{*}}^{3}\right\}\right]^{-1}
$$

then we have

1. $\|\widehat{\boldsymbol{\Omega}}-\boldsymbol{\Omega}\|_{\max } \leq 2 \kappa_{\boldsymbol{\Gamma}^{*}}\left(\left\|\widehat{\boldsymbol{\Sigma}}^{\text {plug }}-\boldsymbol{\Sigma}\right\|_{\max }+8 \alpha^{-1} \delta\right) \leq 2 \kappa_{\boldsymbol{\Gamma}^{*}}\left(1+8 \alpha^{-1}\right) \delta$,

2. $\|\widehat{\boldsymbol{\Omega}}-\boldsymbol{\Omega}\|_{2} \leq 2 \kappa_{\boldsymbol{\Gamma}^{*}}\left(1+8 \alpha^{-1}\right) \min \{\sqrt{s+p}, d\} \delta$,

3. $\|\widehat{\boldsymbol{\Omega}}-\boldsymbol{\Omega}\|_{F} \leq 2 \kappa_{\boldsymbol{\Gamma}^{*}}\left(1+8 \alpha^{-1}\right) \sqrt{s+p} \delta$,

where $\widehat{\boldsymbol{\Omega}}$ is the graphical lasso estimator that solves (13).

We note that $\delta_{n, p}$ corresponds to $\bar{\delta}_{f}\left(n, p^{\tau}\right)$ in the original reference.

CLIME

Let us introduce the class of a precision matrix used in [5]. For $0 \leq q<1$,

$$
\mathcal{U}\left(q, c_{0}(p)\right)=\left\{\boldsymbol{\Omega} \succ 0:\|\boldsymbol{\Omega}\|_{1} \leq M, \max _{1 \leq i \leq p} \sum_{j=1}^{p}\left|\omega_{i j}\right|^{q} \leq s_{0}(p)\right\} .
$$

Theorem (Theorem 6, [5]). If $\|\boldsymbol{\Omega}\|_{1}\left\|\widehat{\mathbf{\Sigma}}^{\text {plug }}-\boldsymbol{\Sigma}\right\|_{\text {max }} \leq \delta$, then we have

1. $\|\widehat{\boldsymbol{\Omega}}-\boldsymbol{\Omega}\|_{\max } \leq 4\|\boldsymbol{\Omega}\|_{1} \delta$,

2. $\|\widehat{\boldsymbol{\Omega}}-\boldsymbol{\Omega}\|_{2} \leq C s_{0}(p)\left(4\|\boldsymbol{\Omega}\|_{1} \delta\right)^{1-q}$, if $\boldsymbol{\Omega} \in \mathcal{U}\left(q, c_{0}(p)\right)$,

3. $\|\widehat{\boldsymbol{\Omega}}-\boldsymbol{\Omega}\|_{F}^{2} / p \leq C s_{0}(p)\left(4\|\boldsymbol{\Omega}\|_{1} \delta\right)^{2-q}$, if $\boldsymbol{\Omega} \in \mathcal{U}\left(q, c_{0}(p)\right)$,

where $\widehat{\boldsymbol{\Omega}}$ is the CLIME estimator that solves (23) and $C>0$ is a numerical constant.

\section{Graphical Dantzig selector}

The graphical Dantzig selector aims to solve $p$ optimization problems below $([48])$

$$
\min _{\beta_{j} \in \mathbb{R}^{p-1}}\left\|\beta_{j}\right\|_{1}, \quad \text { subject to }\left\|\widehat{\boldsymbol{\Sigma}}_{-j, j}^{\text {plug }}-\widehat{\boldsymbol{\Sigma}}_{-j,-j}^{\text {plug }} \beta_{j}\right\|_{\infty} \leq \lambda,
$$

for $j=1, \ldots, p$. Let $d$ be the maximum degree of the graph, or equivalently $d=\max _{i} \sum_{j} \mathrm{I}\left(\left|\omega_{i j}\right| \neq 0\right)$. 
Theorem (A consequence of Lemma 11, [48]). Assume $\boldsymbol{\Omega} \in O(v, \eta, \tau)$ defined by

$$
\begin{array}{r}
O(v, \eta, \tau)=\left\{\boldsymbol{\Omega} \succ 0: v^{-1} \leq \lambda_{\min }(\boldsymbol{\Omega}) \leq \lambda_{\max }(\boldsymbol{\Omega}) \leq v,\|\boldsymbol{\Sigma} \boldsymbol{\Omega}-\mathbf{I}\|_{\max } \leq \eta,\right. \\
\left.\|\boldsymbol{\Omega}\|_{1} \leq \tau\right\} .
\end{array}
$$

If $\tau v\left\|\widehat{\boldsymbol{\Sigma}}^{\text {plug }}-\boldsymbol{\Sigma}\right\| \|_{\max }+\eta v \leq \delta$, then we have

$$
\|\widehat{\mathbf{\Omega}}-\boldsymbol{\Omega}\|_{1} \leq C d \delta
$$

where $\widehat{\boldsymbol{\Omega}}$ is the graphical Dantzig estimator that solves (19) and $C$ depends only on $v, \tau, \lambda_{\min }(\boldsymbol{\Omega}), \lambda_{\max }(\boldsymbol{\Omega})$.

Note that the $\ell_{1}$-norm of a matrix bounds the spectral norm, so we also have

$$
\|\widehat{\boldsymbol{\Omega}}-\mathbf{\Omega}\|_{2} \leq C d \delta
$$

\section{B.4. Proof of Theorem 2}

Proof. Recall that the the proposed estimator when mean is not known has its form as follows: $\widehat{\mathbf{\Sigma}}^{I P W \mu}=\left(\left(\widehat{\boldsymbol{\Sigma}}^{I P W \mu}\right)_{k \ell}, 1 \leq k, \ell \leq p\right)$ with

$$
\left(\widehat{\boldsymbol{\Sigma}}^{I P W \mu}\right)_{k \ell}=\frac{\sum_{i=1}^{n} \tilde{Y}_{i k} \tilde{Y}_{i \ell}}{n \pi_{k \ell}}-\frac{\sum_{i \neq j}^{n} \tilde{Y}_{i k} \tilde{Y}_{j \ell}}{n(n-1) \pi_{k} \pi_{\ell}} .
$$

Let $(k, \ell)$ be a dual in $\{1, \ldots, p\} \times\{1, \ldots, p\}$. Using $\tilde{Y}_{i k}=\delta_{i k} X_{i k}+\delta_{i k} \mu_{k}=$ $Y_{i k}+\delta_{i k} \mu_{k}$, we can decompose the first term in (9) as follows.

$$
\begin{aligned}
& \frac{\sum_{i=1}^{n} \tilde{Y}_{i k} \tilde{Y}_{i \ell}}{n \pi_{k \ell}}-\left(\sigma_{k \ell}+\mu_{k} \mu_{\ell}\right) \\
= & \left\{\frac{\sum_{i=1}^{n} Y_{i k} Y_{i \ell}}{n \pi_{k \ell}}-\sigma_{k \ell}\right\}+\left\{\frac{\sum_{i=1}^{n} \delta_{i k} \delta_{i \ell} \mu_{k} X_{i \ell}}{n \pi_{k \ell}}\right\} \\
& +\left\{\frac{\sum_{i=1}^{n} \delta_{i k} \delta_{i \ell} X_{i k} \mu_{\ell}}{n \pi_{k \ell}}\right\}+\left\{\frac{\sum_{i=1}^{n} \delta_{i k} \delta_{i \ell} \mu_{k} \mu_{\ell}}{n \pi_{k \ell}}-\mu_{k} \mu_{\ell}\right\} \\
= & A_{1}+A_{2}+A_{3}+A_{4} .
\end{aligned}
$$

A deviation inequality for $A_{1}$ comes from Lemma 1 . On the other hands, since $A_{2}, A_{3}$, and $A_{4}$ are independent sum of sub-Gaussian variables, the related concentration inequalities can be found in Lemma 4 (a) and 9. The second term 
in (9) can be decomposed by

$$
\begin{aligned}
& \frac{\sum_{i \neq j} \tilde{Y}_{i k} \tilde{Y}_{j \ell}}{n(n-1) \pi_{k} \pi_{\ell}}-\mu_{k} \mu_{\ell} \\
= & \frac{\sum_{i \neq j}\left(\tilde{Y}_{i k}-\mathbb{E} \tilde{Y}_{i k}\right)\left(\tilde{Y}_{j \ell}-\mathbb{E} \tilde{Y}_{j \ell}\right)}{n(n-1) \pi_{k} \pi_{\ell}}+\frac{\sum_{i \neq j}\left(\tilde{Y}_{i k}-\mathbb{E} \tilde{Y}_{i k}\right) \mathbb{E} \tilde{Y}_{j \ell}}{n(n-1) \pi_{k} \pi_{\ell}} \\
= & \frac{\sum_{i \neq j}\left(\tilde{Y}_{i k}-\mathbb{E} \tilde{Y}_{i k}\right)\left(\tilde{Y}_{j \ell}-\mathbb{E} \tilde{Y}_{i \ell}\right) \mathbb{E} \tilde{Y}_{i k}}{n(n-1) \pi_{k} \pi_{\ell}} \\
n(n-1) \pi_{k} \pi_{\ell} & +\frac{\mu_{\ell} \sum_{i=1}^{n}\left(\tilde{Y}_{i k}-\mathbb{E} \tilde{Y}_{i k}\right)}{n \pi_{k}} \\
= & \frac{\sum_{i \neq j}\left(\tilde{Y}_{i k}-\mathbb{E} \tilde{Y}_{i k}\right)\left(\tilde{Y}_{j \ell}-\mathbb{E} \tilde{Y}_{j \ell}\right)}{n(n-1) \pi_{k} \pi_{\ell}}+\frac{\mu_{\ell} \sum_{i=1}^{n}-\delta_{i k} X_{i k}}{n \pi_{\ell}}+\frac{\mu_{\ell} \sum_{i=1}^{n}\left(\delta_{i k}-\pi_{k}\right)}{n \pi_{k}} \\
& +\frac{\mu_{k} \sum_{i=1}^{n} \delta_{i \ell} X_{i \ell}}{n \pi_{\ell}}+\frac{\mu_{k} \sum_{i=1}^{n}\left(\delta_{i \ell}-\pi_{\ell}\right)}{n \pi_{\ell}} \\
= & B_{1}+B_{2}+B_{3}+B_{4}+B_{5} .
\end{aligned}
$$

The concentration of each term except $B_{1}$ is easily derived using Lemma 4 (a) and 9. To analyze the concentration of $B_{1}$ which is a dependent sum of crossproduct of sub-Gaussian variables, we need a new version of Hanson-Wright inequality. Lemma 5 is more general than that given in [38] in the sense that two random variables $X_{i}, Y_{i}$ are not necessarily equal. The generalization is possible because of the decoupling technique from which we can separately handle $\left\{X_{i}\right.$ : $i \in \Lambda\}$ and $\left\{Y_{i}: i \notin \Lambda\right\}$ for some $\Lambda \subset\{1, \ldots, n\}$. Details of the proof of Lemma 5 can be found in Section B.5.

Lemma 5. Let $(X, Y)$ be a pair of (possibly correlated) random variables satisfying $\mathbb{E} X=\mathbb{E} Y=0$, and

$$
\sup _{r \geq 1} \frac{\left\{\mathbb{E}|X|^{r}\right\}^{1 / r}}{\sqrt{r}} \leq K_{X}, \quad \sup _{r \geq 1} \frac{\left\{\mathbb{E}|Y|^{r}\right\}^{1 / r}}{\sqrt{r}} \leq K_{Y}
$$

Assume $n$ copies $\left\{\left(X_{i}, Y_{i}\right)\right\}_{i=1}^{n}$ of $(X, Y)$ are independently observed. For a matrix $\boldsymbol{A}=\left(a_{i j}, 1 \leq i, j \leq n\right)$ with zero diagonals $a_{i i}=0$, we have that

$\mathrm{P}\left[\left|\sum_{i \neq j} a_{i j} X_{i} Y_{j}\right|>t\right] \leq 2 \exp \left\{-c \min \left(\frac{t^{2}}{K_{X}^{2} K_{Y}^{2}\|\boldsymbol{A}\|_{F}^{2}}, \frac{t}{K_{X} K_{Y}\|\boldsymbol{A}\|_{2}}\right)\right\}, \quad t \geq 0$.

for some numerical constant $c>0$.

Now, we get the concentration bound for $B_{1}$ using the lemma above. Note that the matrix in $\mathbb{R}^{n \times n}$ with off-diagonals 1 and diagonals 0 has both Frobenius 
and spectral norms bounded above by $n$.

$$
\begin{aligned}
\mathrm{P}\left[\mid \sum_{i \neq j}\left(\tilde{Y}_{i k}-\mathbb{E} \tilde{Y}_{i k}\right)\left(\tilde{Y}_{j \ell}-\right.\right. & \left.\left.\mathbb{E} \tilde{Y}_{j \ell}\right) \mid>t\right] \\
& \leq 2 \exp \left\{-c \min \left(\frac{t^{2}}{\sigma_{k k} \sigma_{\ell \ell} K^{4} n^{2}}, \frac{t}{\sigma_{k k}^{1 / 2} \sigma_{\ell \ell}^{1 / 2} K^{2} n}\right)\right\}
\end{aligned}
$$

for $t>0$. Equivalently, it holds

$$
\mathrm{P}\left[\left|\frac{\sum_{i \neq j}\left(\tilde{Y}_{i k}-\mathbb{E} \tilde{Y}_{i k}\right)\left(\tilde{Y}_{j \ell}-\mathbb{E} \tilde{Y}_{j \ell}\right)}{n(n-1) \pi_{k} \pi_{\ell}}\right|>\frac{t \sigma_{k k}^{1 / 2} \sigma_{\ell \ell}^{1 / 2} K^{2}}{(n-1) \pi_{k} \pi_{\ell}}\right] \leq 2 \exp \left\{-c \min \left(t^{2}, t\right)\right\},
$$

Thus, based on the union argument, we get

$$
\begin{aligned}
\mathrm{P}\left[\max _{k, \ell}\left|\frac{\sum_{i \neq j}\left(\tilde{Y}_{i k}-\mathbb{E} \tilde{Y}_{i k}\right)\left(\tilde{Y}_{j \ell}-\mathbb{E} \tilde{Y}_{j \ell}\right)}{n(n-1) \pi_{k} \pi_{\ell}}\right|\right. & \left.>\frac{t \sigma_{\max } K^{2}}{(n-1) \pi_{\min , d}^{2}}\right] \\
& \leq 2 p^{2} \exp \left\{-c \min \left(t^{2}, t\right)\right\}
\end{aligned}
$$

If $\log p>\tilde{c}$ for some $\tilde{c}>1$, then

$$
\mathrm{P}\left[\max _{k, \ell}\left|\frac{\sum_{i \neq j}\left(\tilde{Y}_{i k}-\mathbb{E} \tilde{Y}_{i k}\right)\left(\tilde{Y}_{j \ell}-\mathbb{E} \tilde{Y}_{j \ell}\right)}{n(n-1) \pi_{k} \pi_{\ell}}\right|>\frac{C \sigma_{\max } K^{2}}{\pi_{\min , d}^{2}} \frac{\sqrt{\log p}}{n-1}\right] \leq 2 / p,
$$

for some constant $C>0$, which is dominated by the aimed error rate $\sqrt{\log p / n}$.

Combining all results for $A_{1}, \ldots, A_{4}, B_{1}, \ldots, B_{5}$, we can derive the concentration inequality for each component of $\widehat{\Sigma}^{I P W \mu}$, which completes the proof.

\section{B.5. Proof of Lemma 5}

Proof. Without loss of generality, we assume $K_{X}=K_{Y}=1$. Let $\left\{\eta_{i}\right\}_{i=1}^{n}$ be independent Bernoulli variables with success probability $1 / 2$. Then, by observing $\mathbb{E} \eta_{i}\left(1-\eta_{j}\right)=\mathrm{I}(i \neq j) / 4$, it can be seen that $S \equiv \sum_{i \neq j} a_{i j} X_{i} Y_{j}=4 \mathbb{E}_{\left\{\eta_{i}\right\}} S_{\eta}$ where $S_{\eta}=\sum_{i, j} \eta_{i}\left(1-\eta_{j}\right) a_{i j} X_{i} Y_{j}$ and $\mathbb{E}_{\left\{\eta_{i}\right\}}$ is an expectation taken over $\left\{\eta_{i}\right\}$. Let $\Lambda_{\eta}=\left\{i: \eta_{i}=1\right\}$ be the index set of successes. Since $S_{\eta}=\sum_{i \in \Lambda_{\eta}, j \in \Lambda_{\eta}^{c}} a_{i j} X_{i} Y_{j}$ is a function of $\left\{Y_{j}: j \in \Lambda_{\eta}^{c}\right\}$ given $\left\{\eta_{i}\right\}$ and $\left\{X_{i}: i \in \Lambda_{\eta}\right\}, S_{\eta}$ conditionally follows is a sub-Gaussian distribution.

We assume $\left\{\eta_{i}\right\}$ is conditioned on all the following statements unless specified otherwise. Then, the previous results yield

$$
\begin{aligned}
& \mathbb{E}_{\left\{\left(X_{j}, Y_{j}\right): j \in \Lambda_{\eta}^{c}\right\}}\left[\exp \left(4 \lambda S_{\eta}\right) \mid\left\{X_{i}: i \in \Lambda_{\eta}\right\}\right] \\
& =\mathbb{E}_{\left\{Y_{j}: j \in \Lambda_{\eta}^{c}\right\}}\left[\exp \left(4 \lambda S_{\eta}\right) \mid\left\{X_{i}: i \in \Lambda_{\eta}\right\}\right] \leq \exp \left\{c \lambda^{2} \sum_{j \in \Lambda_{\eta}^{c}}\left(\sum_{i \in \Lambda_{\eta}} a_{i j} X_{i}\right)^{2}\right\}
\end{aligned}
$$

where the equality holds since $\exp \left(4 \lambda S_{\eta}\right)$ does not depend on $\left\{X_{j}\right\}_{j \in \Lambda_{\eta}^{c}}$ and the inequality is from sub-Gaussianity of $S_{\eta}$. Taking expectation with respect 
to $\left\{X_{i}: i \in \Lambda_{\eta}\right\}$ on both sides, we get the following result;

$$
\begin{aligned}
& \mathbb{E}_{\left\{X_{i}: i \in \Lambda_{\eta}\right\},\left\{\left(X_{j}, Y_{j}\right): j \in \Lambda_{\eta}^{c}\right\}}\left[\exp \left(4 \lambda S_{\eta}\right)\right] \\
& \leq \mathbb{E}_{\left\{X_{i}: i \in \Lambda_{\eta}\right\}}\left[\exp \left\{c \lambda^{2} \sum_{j \in \Lambda_{\eta}^{c}}\left(\sum_{i \in \Lambda_{\eta}} a_{i j} X_{i}\right)^{2}\right\}\right] \\
& =\mathbb{E}_{\left\{X_{i}\right\}}\left[\exp \left\{c \lambda^{2} \sum_{j \in \Lambda_{\eta}^{c}}\left(\sum_{i \in \Lambda_{\eta}} a_{i j} X_{i}\right)^{2}\right\}\right],
\end{aligned}
$$

where the equality holds from independence among $n$ samples. Also, since the left-hand side does not depend on $\left\{Y_{i}: i \in \Lambda_{\eta}\right\}$, we get

$\mathbb{E}_{\left\{\left(X_{i}, Y_{i}\right)\right\}}\left[\exp \left(4 \lambda S_{\eta}\right) \mid\left\{\eta_{i}\right\}\right] \leq \mathbb{E}_{\left\{X_{i}\right\}}\left[\exp \left\{c \lambda^{2} \sum_{j \in \Lambda_{\eta}^{c}}\left(\sum_{i \in \Lambda_{\eta}} a_{i j} X_{i}\right)^{2}\right\} \mid\left\{\eta_{i}\right\}\right]\left(\equiv T_{\eta}\right)$,

where we begin to display the conditional dependency on $\left\{\eta_{i}\right\}$. Following the step 3 and 4 in [38], we can achieve an uniform bound of $T_{\eta}$ independent of $\left\{\eta_{i}\right\}$ and thus get

$$
T_{\eta} \leq \exp \left\{C \lambda^{2}\|A\|_{F}^{2}\right\} \quad \text { for } \lambda \leq c /\|A\|_{2},
$$

for some positive constants $c$ and $C$. Then, we have

$$
\begin{aligned}
\mathbb{E}[\exp (\lambda S)] & =\mathbb{E}\left[\exp \left(\mathbb{E}_{\left\{\eta_{i}\right\}} 4 \lambda S_{\eta}\right)\right] \\
& \leq \mathbb{E}_{\left\{\left(X_{i}, Y_{i}\right)\right\}_{i},\left\{\eta_{i}\right\}}\left[\exp \left(4 \lambda S_{\eta}\right)\right](\because \text { Jensen's inequality }) \\
& =\mathbb{E}\left[\mathbb{E}\left[\exp \left(4 \lambda S_{\eta}\right) \mid\left\{\eta_{i}\right\}\right]\right] \\
& =\mathbb{E}\left[T_{\eta}\right] \leq \exp \left\{C \lambda^{2}\|A\|_{F}^{2}\right\} \quad \text { for } \lambda \leq c /\|A\|_{2},
\end{aligned}
$$

Following the step 5 in [38], we can get the concentration of $S$ given in the lemma below. Let $\|X\|_{\psi_{2}}$ be a $\psi_{2}$-norm of $X$ defined by

$$
\|X\|_{\psi_{2}}=\inf \left\{R>0: \mathbb{E} e^{\frac{|X|^{2}}{R^{2}}} \leq 2\right\} .
$$

Lemma 6. Let $(X, Y)$ be a pair of (possibly correlated) random variables satisfying $\mathbb{E} X=\mathbb{E} Y=0$, and

$$
\|X\|_{\psi_{2}} \leq K_{X},\|Y\|_{\psi_{2}} \leq K_{Y}
$$

Assume $n$ samples $\left\{\left(X_{i}, Y_{i}\right)\right\}_{i=1}^{n}$ are identically and independently observed. For a matrix $A=\left(a_{i j}, 1 \leq i, j \leq n\right)$ with zero diagonals, we have that

$\mathrm{P}\left[\left|\sum_{i \neq j} a_{i j} X_{i} Y_{j}\right|>t\right] \leq 2 \exp \left\{-c \min \left(\frac{t^{2}}{K_{X}^{2} K_{Y}^{2}\|A\|_{F}^{2}}, \frac{t}{K_{X} K_{Y}\|A\|_{2}}\right)\right\}, \quad t \geq 0$.

for some numerical constant $c>0$.

Note that the finite $\psi_{2}$-norm in (21) characterizes a sub-Gaussian random variable and can be replaced by the uniformly bounded moments in (17), since $\sup _{r \geq 1}\left\{\mathbb{E}|X|^{r}\right\}^{1 / r} / \sqrt{r} \leq K$ implies $\|X\|_{\psi_{2}} \leq 2 e K$. In other words, provided $X_{i}$ and $\bar{Y}_{j}$ satisfy the moment condition with constants $K_{X}$ and $K_{Y}$, respectively, the conclusion of the lemma above still holds (with different $c$ ). This completes the proof. 


\section{B.6. Proof of Theorem 3}

Theorem 3 is not difficult to show if Lemmas 1, 7, and 8 are used together. Let us show and prove the two additional lemmas. First, the following lemma shows how the concentration of (10) is related to that of $\hat{\pi}_{j k}$.

Lemma 7. Assume

$$
\begin{aligned}
& \max _{k, \ell}\left|1 / \pi_{k \ell}-1 / \hat{\pi}_{k \ell}\right|<B_{1}, \quad \hat{\pi}_{k \ell}>0, \forall k, \ell, \\
& \left\|\boldsymbol{S}_{Y}-\boldsymbol{\Sigma}^{\pi}||_{\text {max }}<B_{2}, \quad\right\| \widehat{\boldsymbol{\Sigma}}^{I P W}-\left.\boldsymbol{\Sigma}\right|_{\text {max }}<B_{3}
\end{aligned}
$$

where $B_{1}, B_{2}$, and $B_{3}$ are positive constants. Then, we have

$$
\| \widehat{\boldsymbol{\Sigma}}^{I P W \pi}-\mathbf{\Sigma}||_{\max } \leq B_{1} B_{2}+B_{1} \sigma_{\max }+B_{3} .
$$

Proof. By the triangular inequality, we observe

$$
\begin{aligned}
& \left\|\widehat{\boldsymbol{\Sigma}}^{I P W \pi}-\boldsymbol{\Sigma}\right\|_{\max } \leq\left\|\widehat{\boldsymbol{\Sigma}}^{I P W \pi}-\widehat{\boldsymbol{\Sigma}}^{I P W}\right\|_{\max }+\left\|\widehat{\boldsymbol{\Sigma}}^{I P W}-\mathbf{\Sigma}\right\| \\
& \leq \max _{k, \ell}\left|1 / \pi_{k \ell}-1 / \hat{\pi}_{k \ell}\right| \cdot\left\|\boldsymbol{S}_{Y}\right\|_{\max }+\left\|\widehat{\boldsymbol{\Sigma}}^{\text {max }}-\boldsymbol{\Sigma}\right\|_{\max } \\
& \leq \max _{k, \ell}\left|1 / \pi_{k \ell}-1 / \hat{\pi}_{k \ell}\right| \cdot|| \boldsymbol{S}_{Y}-\boldsymbol{\Sigma}^{\pi} \|_{\max } \\
& +\max _{k, \ell}\left|1 / \pi_{k \ell}-1 / \hat{\pi}_{k \ell}\right| \cdot|| \boldsymbol{\Sigma}^{\pi} \|_{\max } \\
& +\| \widehat{\boldsymbol{\Sigma}}^{I P W}-\left.\mathbf{\Sigma}\right|_{\max }
\end{aligned}
$$

where $\boldsymbol{S}_{Y}=n^{-1} \sum_{i=1}^{n} Y_{i} Y_{i}^{\mathrm{T}}$ and $\boldsymbol{\Sigma}^{\pi}=\left(\pi_{j k} \sigma_{j k}, 1 \leq j, k \leq p\right)$. Thus, we get

$$
\left\|\widehat{\boldsymbol{\Sigma}}^{I P W \pi}-\mathbf{\Sigma}\right\|_{\max } \leq B_{1} B_{2}+B_{1}\left\|\boldsymbol{\Sigma}^{\pi}\right\|_{\max }+B_{3}
$$

Finally, we note that

$$
\left\|\Sigma^{\pi}\right\|_{\max } \leq\|\boldsymbol{\Sigma}\|_{\max }=\sigma_{\max }
$$

where the last equality holds for a symmetric positive definite matrix.

Lemma 8. Assume the sample size and dimension satisfy $n / \log p>c / \pi_{\min }^{2}$ for some numerical constant $C>0$. Then, with probability at least $1-1 / p$

$$
\max _{k, \ell}\left|\frac{1}{\pi_{k \ell}}-\frac{1}{\hat{\pi}_{k \ell}^{e m p}}\right| \leq \frac{C}{\pi_{m i n}^{2}} \sqrt{\frac{\log p}{n}} \text { and } \hat{\pi}_{k \ell}^{e m p}>0, \forall k, \ell .
$$

Proof. First, we observe that on the event $G=\cap_{k, \ell} G_{k, \ell}$ where $G_{k, \ell}=\left\{\hat{\pi}_{k \ell}^{e m p}>\right.$ $0\}$, we have for $t>0$

$$
\left|1 / \pi_{k \ell}-1 / \hat{\pi}_{k \ell}^{e m p}\right| \leq t \Leftrightarrow\left(1-t \pi_{k \ell}\right) \hat{\pi}_{k \ell}^{e m p} \leq \pi_{k \ell} \text { and }\left(1+t \pi_{k \ell}\right) \hat{\pi}_{k \ell}^{e m p} \geq \pi_{k \ell} .
$$

Note that for $t>1 / \pi_{k \ell},\left(1-t \pi_{k \ell}\right) \hat{\pi}_{k \ell}^{e m p} \leq \pi_{k \ell}$ always holds. Let $B_{k \ell}=\{(1+$ $\left.\left.t \pi_{k \ell}\right) \hat{\pi}_{k \ell}^{e m p} \geq \pi_{k \ell}\right\}$. Then, we get

$$
\mathrm{P}\left[\left\{\left|1 / \pi_{k \ell}-1 / \hat{\pi}_{k \ell}^{e m p}\right| \leq t\right\} \cap\left\{\hat{\pi}_{k \ell}^{e m p}>0\right\}\right]=\mathrm{P}\left(B_{k \ell} \cap G_{k, \ell}\right)=\mathrm{P}\left(B_{k \ell}\right), \quad t>1 / \pi_{k \ell}
$$

To compute the last probability, we use the deviation inequality for a sum of bounded random variables. 
Lemma 9. Let $X_{i}$ be an independent bounded random variable in $\left[a_{i}, b_{i}\right]$ with $\mathbb{E} X_{i}=\mu_{i}, i=1, \ldots, n$. Then, there exists a numerical constant $C>0$ such that for $t>0$,

$$
\mathrm{P}\left[ \pm \sum_{i=1}^{n}\left(X_{i}-\mu_{i}\right) \geq t\right] \leq \exp \left\{-\frac{C t^{2}}{\sum_{i=1}^{n}\left(b_{i}-a_{i}\right)^{2}}\right\} .
$$

By using Lemma 9, it holds

$$
\mathrm{P}\left(B_{k \ell}^{c}\right)=\mathrm{P}\left[\hat{\pi}_{k \ell}^{e m p}-\pi_{k \ell}<-\frac{t \pi_{k \ell}^{2}}{1+t \pi_{k \ell}}\right] \leq \exp \left\{-\frac{C n t^{2} \pi_{k \ell}^{4}}{\left(1+t \pi_{k \ell}\right)^{2}}\right\} .
$$

We define $t^{*}>0$ by the solution of

$$
\frac{\left(t^{*}\right)^{2} \pi_{k \ell}^{4}}{\left(1+t^{*} \pi_{k \ell}\right)^{2}}=\frac{3 \log p}{C n} .
$$

Then, a simple calculation shows that if $3 \log p /(C n)<\pi_{k \ell}^{2} / 4$

$$
t^{*}>1 / \pi_{k \ell}, \quad t^{*}<\frac{\tilde{C}}{\pi_{k \ell}^{2}} \sqrt{\frac{\log p}{n}}
$$

for some numerical constant $\tilde{C}>0$. Hence, replacing $t$ by $t^{*}$, we can conclude that

$$
\begin{aligned}
& \mathrm{P}\left[\left\{\left|1 / \pi_{k \ell}-1 / \hat{\pi}_{k \ell}^{e m p}\right| \leq \frac{\tilde{C}}{\pi_{k \ell}^{2}} \sqrt{\frac{\log p}{n}}\right\} \cap G_{k, \ell}\right] \\
& =\mathrm{P}\left[\hat{\pi}_{k \ell}^{e m p}-\pi_{k \ell} \geq-\frac{t^{*} \pi_{k \ell}^{2}}{1+t^{*} \pi_{k \ell}}\right] \\
& \geq 1-1 / p^{3}
\end{aligned}
$$

for $n / \log p>c / \pi_{k \ell}^{2}$ with a numerical constant $c>0$. Therefore,

$$
\begin{aligned}
& \mathrm{P}\left[\left\{\max _{k, \ell}\left|1 / \pi_{k \ell}-1 / \hat{\pi}_{k \ell}^{e m p}\right| \leq \frac{\tilde{C}}{\pi_{\min }^{2}} \sqrt{\frac{\log p}{n}}\right\} \cap G\right] \\
& \geq 1-\sum_{k, \ell} \mathrm{P}\left[\left\{\left|1 / \pi_{k \ell}-1 / \hat{\pi}_{k \ell}^{e m p}\right| \leq \frac{\tilde{C}}{\pi_{k \ell}^{2}} \sqrt{\frac{\log p}{n}}\right\}^{c} \cup G_{k, \ell}^{c}\right] \\
& \geq 1-1 / p
\end{aligned}
$$

for $n / \log p>c / \pi_{\min }^{2}$.

\section{Appendix C: Additional analyses and details in Section 4}

\section{C.1. Non-PSD input for CLIME}

In what follows, we distinguish between a plug-in matrix (estimator) $\widehat{\boldsymbol{\Sigma}}^{\text {plug }}$ and an initial matrix (estimator) $\boldsymbol{\Sigma}^{(0)}$ (or $\boldsymbol{\Omega}^{(0)}$ ) that is used to initialize iterative steps. 
We analyze the CLIME method proposed by [5], which solves

$$
\min _{\boldsymbol{\Omega} \in \mathbb{R}^{p \times p}}|\boldsymbol{\Omega}|_{1} \quad \text { s.t. } \quad \| \widehat{\boldsymbol{\Sigma}}^{\text {plug }} \boldsymbol{\Omega}-\mathbf{I}||_{\max } \leq \lambda .
$$

[5] divide (23) into $p$ column-wise problems and relax each problem to be a linear programming, which leads to Algorithm 2.

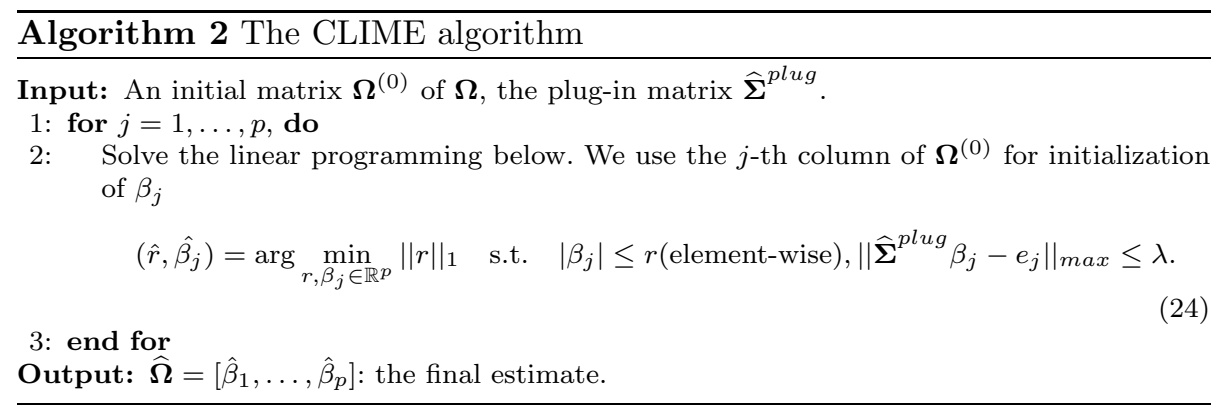

It is easily seen that the optimization problem (23) is convex regardless of the plug-in matrix. Moreover, Algorithm 2 does not require any constraint in the two inputs for a well-defined solution, contrary to Algorithm 1. However, the current implementations (e.g. clime version 0.4.1 from [5], fastclime version 1.4.1 from [30]) set the initial by solving $\boldsymbol{\Omega}^{(0)}\left(\widehat{\boldsymbol{\Sigma}}^{\text {plug }}+\lambda \mathbf{I}\right)=\mathbf{I}$, which is not applicable to our case since an initialization from $\boldsymbol{\Omega}^{(0)}\left(\widehat{\boldsymbol{\Sigma}}^{I P W}+\lambda \mathbf{I}\right)=\mathbf{I}$ is not well-posed unless $\widehat{\boldsymbol{\Sigma}}^{I P W}+\lambda \mathbf{I}$ is positive definite. [21] also point out that the solution of (23) may not exist, unless an input matrix $\widehat{\boldsymbol{\Sigma}}^{\text {plug }}$ is guaranteed to be PSD. We conjecture this irregularity is due to the initialization. Thus, our proposal for the inputs is

$$
\widehat{\boldsymbol{\Sigma}}^{\text {plug }} \leftarrow \widehat{\boldsymbol{\Sigma}}^{I P W}, \quad \boldsymbol{\Omega}^{(0)} \leftarrow \operatorname{diag}\left(\widehat{\boldsymbol{\Sigma}}^{I P W}\right)^{-1} .
$$

Similarly to the graphical lasso, one should modify the implemented $\mathrm{R}$ functions (e.g. clime in clime package) to separately handle two inputs, since it is not allowed for now to control two input matrices $\boldsymbol{\Omega}^{(0)}$ and $\widehat{\boldsymbol{\Sigma}}^{\text {plug }}$ independently.

\section{C.2. Failure of Algorithm 1 under missing data}

It is mentioned that the undesirable property, non-PSDness, of the IPW estimator may hamper downstream multivariate procedures. We give one of the examples where it causes a problem; the graphical lasso. Recall that the existing algorithms available in glasso and huge packages are not suitable especially with the tuning parameter fixed at small $\lambda$, since they use the non-PSD initial matrix $\boldsymbol{\Sigma}^{(0)}=\widehat{\boldsymbol{\Sigma}}^{I P W}+\lambda \mathbf{I}$. As a consequence, in Figure 4 where data is similarly generated to the simulation study (see Section D), the blue solid ROC 
curves end at FPR values far less than 1 when the coordinate descent algorithm provided in huge is used. On the contrary, the QUIC algorithm (red dashed) returns a full length of ROC curves. It is noted that since the graphical lasso has a unique solution, two algorithms create the same path, as long as convergence is reached.

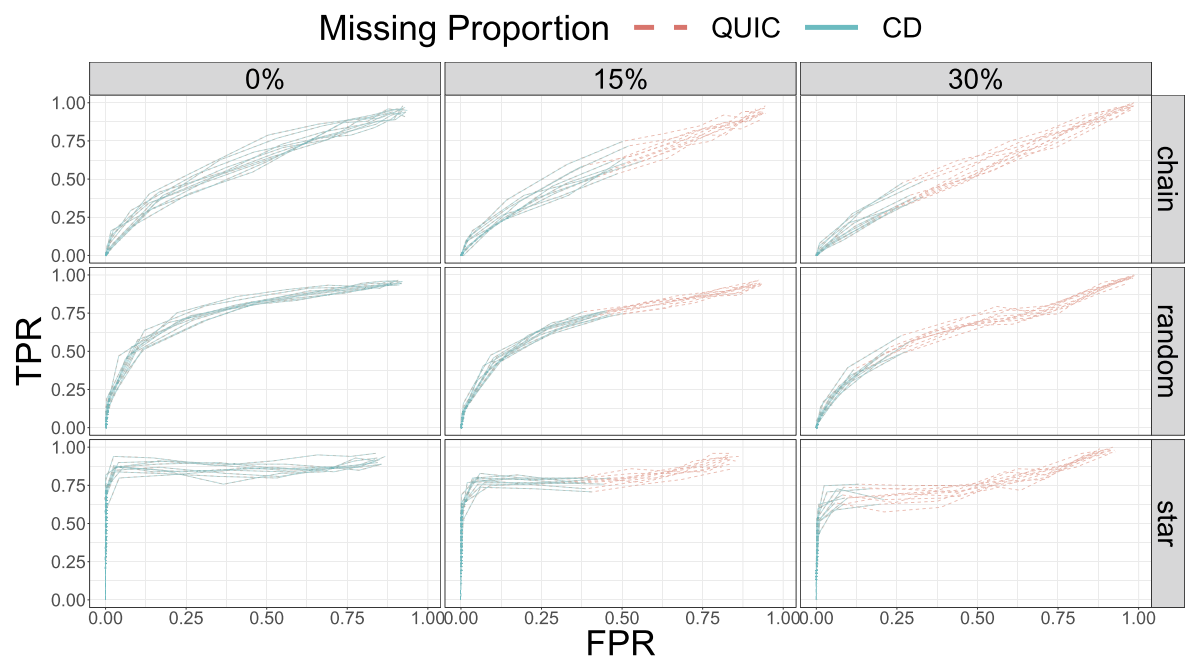

FIG 4. Comparison of ROC curves between two different algorithms for solving the graphical lasso using incomplete data. Here, $n=100, r=1$, and the dependent missing structure are assumed. The oracle IPW estimator is plugged-in. We randomly generate 10 data sets.

\section{Appendix D: Additional analyses and details in Section 5}

\section{D.1. Details of the simulation setting}

Recall that we generate Gaussian random vectors $X_{i}, i=1, \ldots, n$, in $\mathbb{R}^{p}$ with mean vector 0 and precision matrix $\boldsymbol{\Omega}=\left(\omega_{i j}, 1 \leq i, j \leq p\right)$ under different pairs of $n=50,100,200$ and $p$ satisfying $r(=p / n)=0.2,1,2$. The graph structure induced by a precision matrix and the missing structure are described in details below.

Graph structure (precision matrix)

1. Chain-structured graph: The edge set $E$ of a graph is defined by the structure of a chain graph. $\omega_{i j}=0.1$, if $(i, j) \in E$, and 0, otherwise; $\omega_{i i}=1$.

2. Star-structured graph: The edge set $E$ of a graph is defined by the structure of a star-shaped graph. $\omega_{i j}=0.9 / \sqrt{p-1}$, if $(i, j) \in E$, and 0 , otherwise; $\omega_{i i}=1$.

\footnotetext{
${ }^{4}$ The off-diagonal element $\omega_{i j}$ should be less than $1 / \sqrt{p-1}$ to satisfy $\Omega \succ 0$.
} 
3. Erdös-Rényi random graph: Each off-diagonal component in the upper part of $\boldsymbol{B}$ is independently generated, and equals to 0.5 with probability $\log p / p$ and 0 otherwise. Then, the lower part of $\boldsymbol{B}$ is filled with the transposed upper part. Finally, some positive constant is added to the diagonals, i.e., $\boldsymbol{\Omega}=\boldsymbol{B}+1.5\left|\lambda_{\min }\right| \mathbf{I}$, to satisfy PDness where $\lambda_{\min }$ is the smallest eigenvalue of $\boldsymbol{B}$.

Every $\boldsymbol{\Omega}$ is rescaled so that the largest eigenvalue of $\boldsymbol{\Omega}$ is set as 1 .

\section{Missing structure}

Two structures are under consideration to impose missing on data. The first structure is the independent structure where every component of $X_{i}$ is independently exposed to missing with equal probability;

$$
\delta_{i k} \sim \operatorname{Ber}\left(\pi^{(1)}\right), \quad k=1, \ldots, p, \text { independently }
$$

where $0<\pi^{(1)}<1$. Another structure is designed to model dependency within missing indicators. We assume missingness in the first half of $p$ components (assume even $p$ here) forces missing values in the other halves. First, we generate $p$ independent missing indicators as before

$$
\tilde{\delta}_{i k} \sim \operatorname{Ber}\left(\pi^{(2)}\right), \quad k=1, \ldots, p, \text { independently, }
$$

for $0<\pi^{(2)}<1$. Then, dependent indicators are defined by

$$
\delta_{i k}=\tilde{\delta}_{i k}, \quad \delta_{i, k+p / 2}=\min \left\{\tilde{\delta}_{i k}, \tilde{\delta}_{i, k+p / 2}\right\}, \quad k=1, \ldots, p / 2 .
$$

Thus, the $(k+p / 2)$-th component cannot be observed unless its pair is observed, or $\delta_{i k}=1(k=1, \ldots, p / 2)$. An average proportion of missing elements is $1-\pi^{(1)}$ for the independent case and $\left(1-\pi^{(2)}\right)\left(2+\pi^{(2)}\right) / 2$ for the dependent case. Consequently, the proportion of missing denoted by $\alpha$ can be tuned by changing $\pi^{(1)}$ or $\pi^{(2)}$. For example, under the dependent missing structure, for $\alpha=0.3$, $\pi^{(2)}$ is uniquely determined by solving the quadratic equation

$$
\left(1-\pi^{(2)}\right)\left(2+\pi^{(2)}\right) / 2=0.3 .
$$

We choose different values $\alpha=0,0.15,0.3$. The case $\alpha=0$ where all samples are completely observed is included as a reference.

\section{Estimators}

Based on our experience, the graphical lasso is preferred to the CLIME in estimation of sparse precision matrices since the implemented $R$ packages are either too conservative to find true edges ( $\mathrm{R}$ package fastclime) or too slow ( $\mathrm{R}$ package clime). We exploit QUIC algorithm proposed by [18] to solve the graphical 
lasso (13). The grid of a tuning parameter $\lambda \in \Lambda$ is defined adaptively to the plug-in matrix $\widehat{\boldsymbol{\Sigma}}^{\text {plug }}$

$$
\Lambda=\{\exp \{\log (\kappa M)-d \log (\kappa) /(T-1)\}: d=0, \ldots, T-1\},
$$

where $0<\kappa<1$ and $M=\left\|\widehat{\boldsymbol{\Sigma}}^{\text {plug }}-\operatorname{diag}\left(\widehat{\boldsymbol{\Sigma}}^{\text {plug }}\right)\right\|_{\text {max }}$. Note that the points in $\Lambda$ are equally spaced in $\log$-scale from $\log (\kappa M)$ to $\log M$ by length of $T$. $\kappa$ is set as 0.1 and $T$ as 10 .

\section{D.2. Additional results of the simulation study}

We investigate the finite sample performance by changing various parameters (e.g. $r=p / n$, missing proportion) in the simulation study. To evaluate estimation accuracy and support recovery of the Gaussian graphical model, different matrix norms and an area under the receiver operating characteristic (ROC) curve are used.

\section{Estimation accuracy}

We numerically examine behaviors of the inverse covariance matrix estimated using the IPW estimator as simulation parameters vary. To this end, the Frobenius and spectral norms are used to measure the accuracy of an estimator. We fix the $\lfloor 0.7 T\rfloor$-th tuning parameter in $\Lambda$ (in an increasing order) to get a single sparse precision matrix, because selection of the tuning parameter is not of our primary interest and our findings stated below do not change much according to the tuning parameter.

Figures 5 and 6 show that the ratio of the sample size and dimension is one of the key factors that determines the magnitude of estimation error. It is uniformly observed that larger size of a precision matrix is more difficult to estimate, but the degree of difficulty depends on the shape of the true graphs (or precision matrix).

Figures 7 and 8 compare the performance of the two plug-in matrices. When complete data is available, no adjustment for missing is needed so that there is no difference in errors (see the leftmost red boxplots in each sub-figure). If missing occurs in data, the precision matrix estimator based on the oracle IPW estimator is closer to the true matrix (either $\boldsymbol{\Sigma}$ or $\boldsymbol{\Omega}$ ), and the extent is more evident as the missing proportion $\alpha$ increases.

Figures 9 and 10 imply that dependency in missing degrades estimation accuracy, as the missing proportion is set at the same level in both missing structures. We do not show the results when using complete data (i.e., $\alpha=0$ ) since the two missing structures are the same by definition.

Support recovery

We investigate the support recovery of the Gaussian graphical model using the ROC curve. It is observed that the ROC curves end at different false positive 

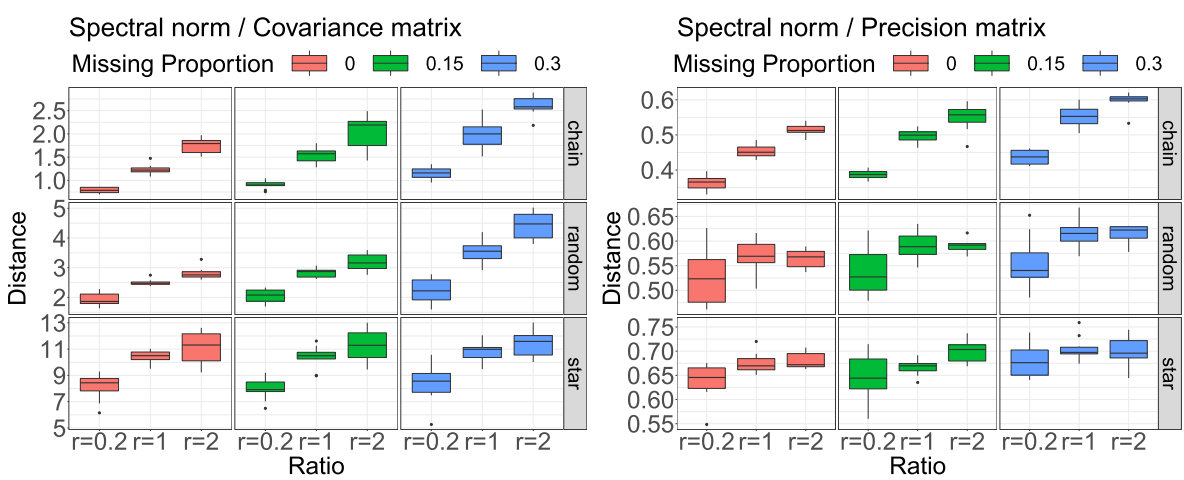

FIG 5. Boxplots of the spectral norm with different ratios $r(=p / n)=0.2,1,2$. Here, the dependent missing structure and $n=100$ are assumed. The oracle IPW estimator is pluggedin. $\left\|\widehat{\boldsymbol{\Omega}}^{-1}-\boldsymbol{\Omega}^{-1}\right\|$ (left) and $\|\widehat{\boldsymbol{\Omega}}-\boldsymbol{\Omega}\|$ (right) are measured.
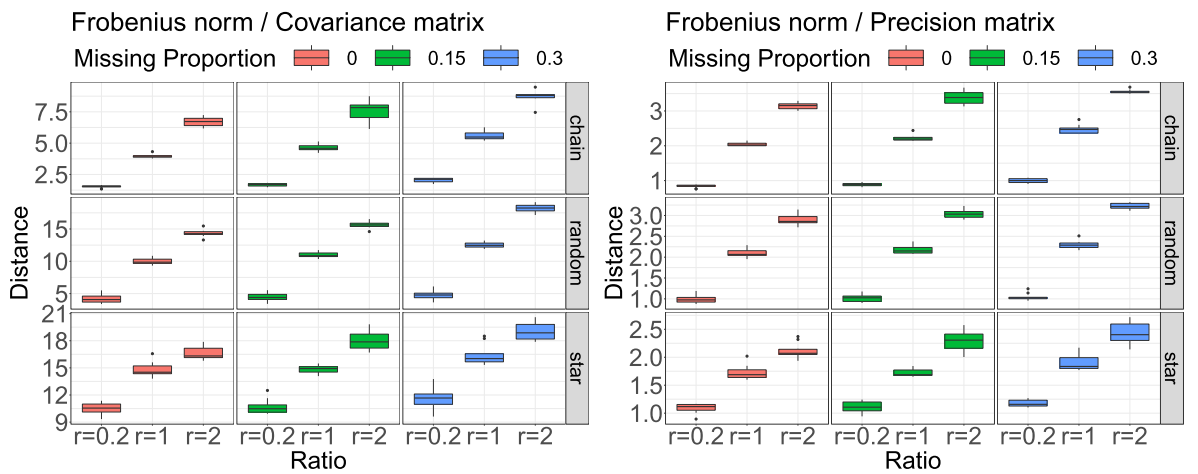

FIG 6. Boxplots of the Frobenius norm with different ratios $r(=p / n)=0.2,1,2$. Here, the dependent missing structure and $n=100$ are assumed. The oracle IPW estimator is pluggedin. $\left\|\widehat{\boldsymbol{\Omega}}^{-1}-\boldsymbol{\Omega}^{-1}\right\|$ (left) and $\|\widehat{\boldsymbol{\Omega}}-\boldsymbol{\Omega}\|$ (right) are measured.

rate (FPR) values, especially when different missing proportions are assumed (see Figure 11).

Thus, it is not fair to directly compare an area under the curve (AUC) because the maximum value of AUC depends on the endpoint (largest value) of FPR and thus cannot reach 1 if the endpoint is less than 1 . Instead, we use the rescaled partial AUC (pAUC) proposed by [45]. The pAUC rescales the AUC by the largest FPR in the ROC curve (see [45] for more details). Then, the rescaled AUCs from different curves that end at different FPR values have the same range $[0,1]$. Figure 12 shows the results of the pAUC as the simulation parameters are varying. Considering a large value of the pAUC implies better performance in the support recovery, we have similar interpretations based on the given results as before. 

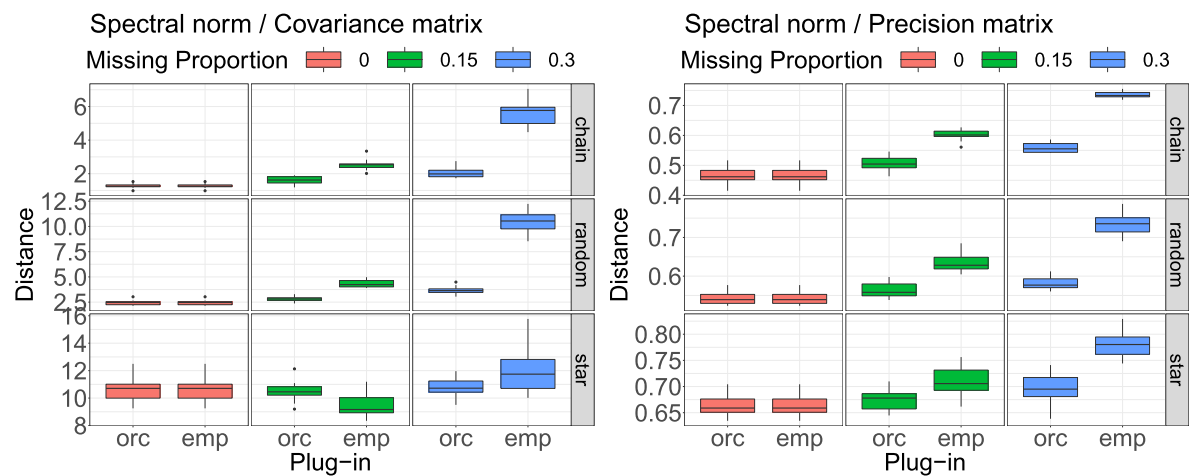

FIG 7. Boxplots of the spectral norm with different plug-in estimators ("emp" and "orc"). Here, the dependent missing structure, $n=100$ and $r=1$ are assumed. $\left\|\widehat{\boldsymbol{\Omega}}^{-1}-\boldsymbol{\Omega}^{-1}\right\|$ (left) and $\|\widehat{\boldsymbol{\Omega}}-\mathbf{\Omega}\|$ (right) are measured.
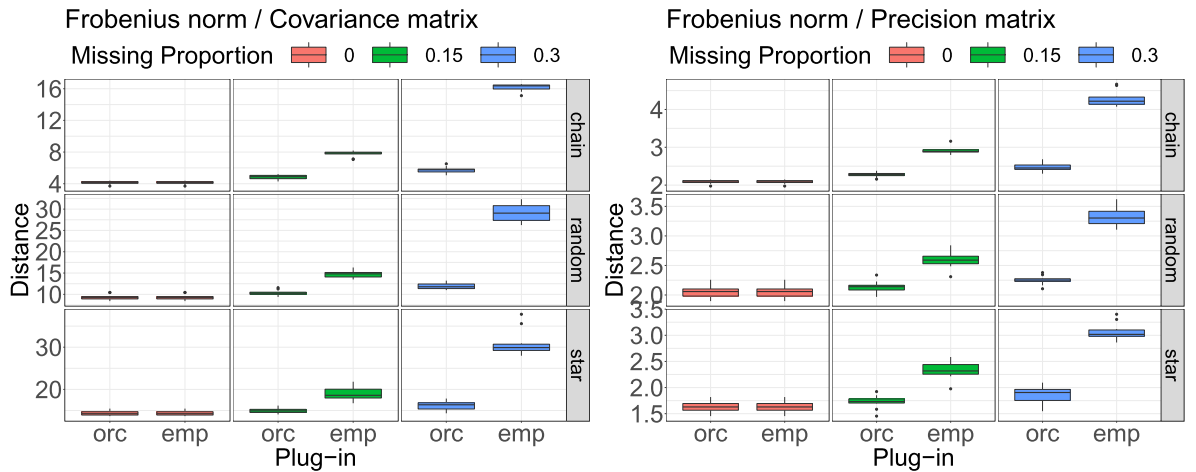

FIG 8. Boxplots of the Frobenius norm with different plug-in estimators ("emp" and "orc"). Here, the dependent missing structure, $n=100$ and $r=1$ are assumed. $\left\|\widehat{\boldsymbol{\Omega}}^{-1}-\boldsymbol{\Omega}^{-1}\right\|$ (left) and $\|\widehat{\boldsymbol{\Omega}}-\boldsymbol{\Omega}\|$ (right) are measured.

\section{Unknown mean}

Continuing from Section 5.4, we measure the performance of the graphical lasso estimator obtained by plugging-in $\widehat{\boldsymbol{\Sigma}}^{I P W \mu}$ in (13). $\|\widehat{\boldsymbol{\Omega}}-\boldsymbol{\Omega}\|$ is computed by the Frobenius and spectral norms and its boxplots are given in Figure 13.

\section{Appendix E: Details in Section 6}

We use the riboflavin data available from the $\mathrm{R}$ package hdi, where 4088 gene expressions are observed across 71 samples. Each variable is log-transformed and then centered. We select 1000 genes with the largest empirical variances for 

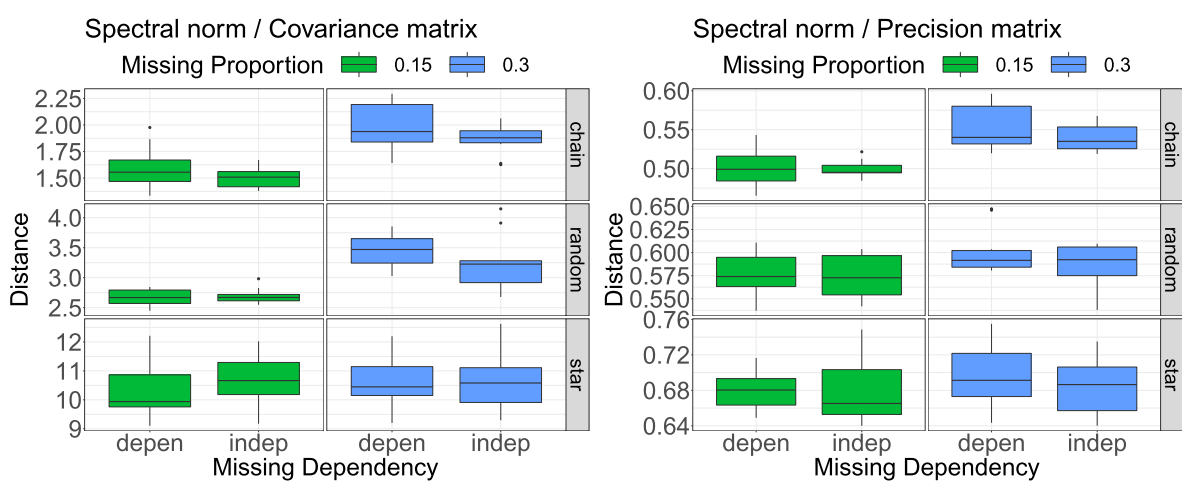

FIG 9. Boxplots of the spectral norm with different missing structures ("depen" and "indep"). Here, $n=100$ and $r=1$ are assumed. The oracle IPW estimator is plugged-in. $\left\|\widehat{\boldsymbol{\Omega}}^{-1}-\boldsymbol{\Omega}^{-1}\right\|$ (left) and $\|\widehat{\boldsymbol{\Omega}}-\boldsymbol{\Omega}\|$ (right) are measured.
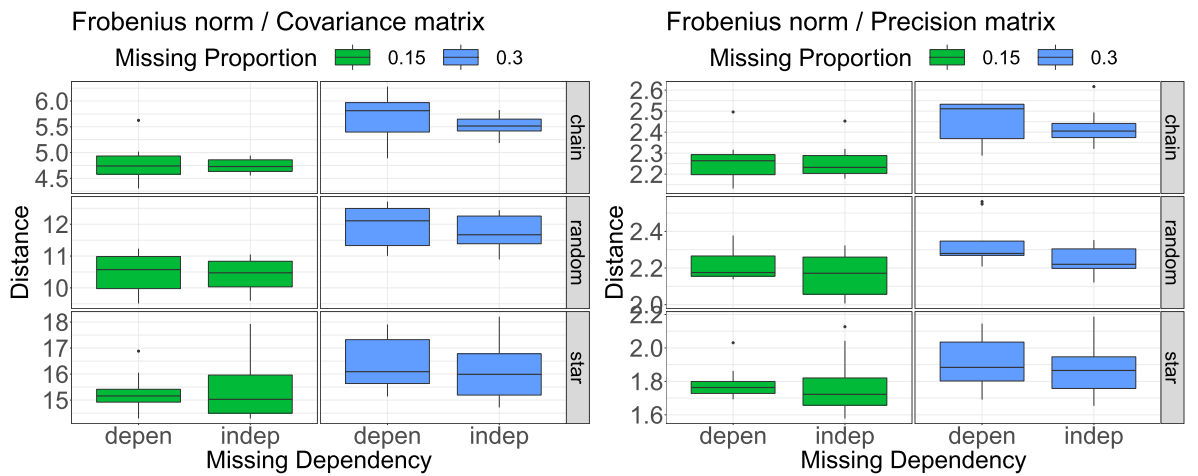

FIG 10. Boxplots of the Frobenius norm with different missing structures ("depen" and "indep"). Here, $n=100$ and $r=1$ are assumed. The oracle IPW estimator is plugged-in. $\left\|\widehat{\boldsymbol{\Omega}}^{-1}-\boldsymbol{\Omega}^{-1}\right\|$ (left) and $\|\widehat{\boldsymbol{\Omega}}-\boldsymbol{\Omega}\|$ (right) are measured.

the sake of simplicity. As in the previous analyses, the QUIC algorithm is used to solve the graphical lasso.

With the complete data set, we solve the graphical lasso (13) with a fixed $\lambda$ and set the obtained estimate $\boldsymbol{\Omega}_{\lambda}$ as the ground truth precision matrix. We generate three different models with $\lambda_{1}<\lambda_{2}<\lambda_{3}$. Note that the estimated precision matrix with a smaller tuning parameter (e.g. $\lambda_{1}$ ) gives a denser true model that has a precision matrix with more non-zero elements. We also consider another ground-truth precision matrix with an optimal tuning parameter that is chosen by the cross-validation procedure, following [23]. Let an index set of $n$ samples split into $K$ folds $\left\{G_{k}\right\}_{k=1}^{K}$ of equal size. Without samples in the $k$-th fold, we estimate the precision matrix at a fixed $\lambda$, denoted by $\boldsymbol{\Omega}_{\lambda}^{(k)}$. We finally choose $\lambda_{C V}$ among a grid of $\lambda$ 's that minimizes the cross-validated (negative) 


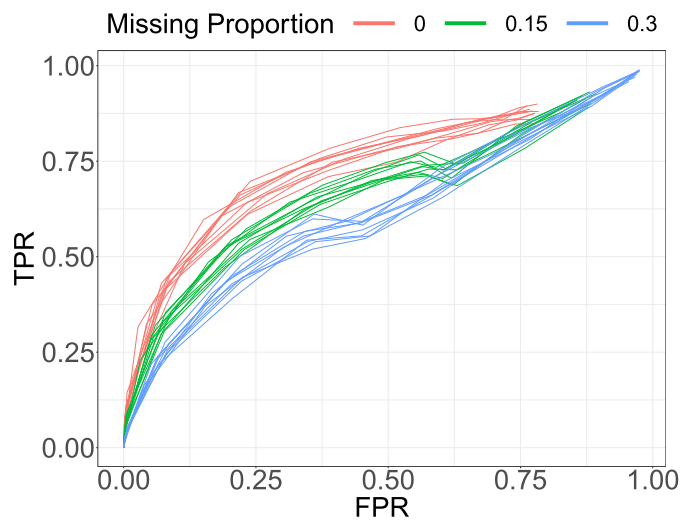

FIG 11. The ROC curves according to different missing proportions with 10 times of repetition. Here, $n=100, r=1$, a random graph structure, and the dependent missing structure are assumed. The oracle IPW estimator is plugged-in.
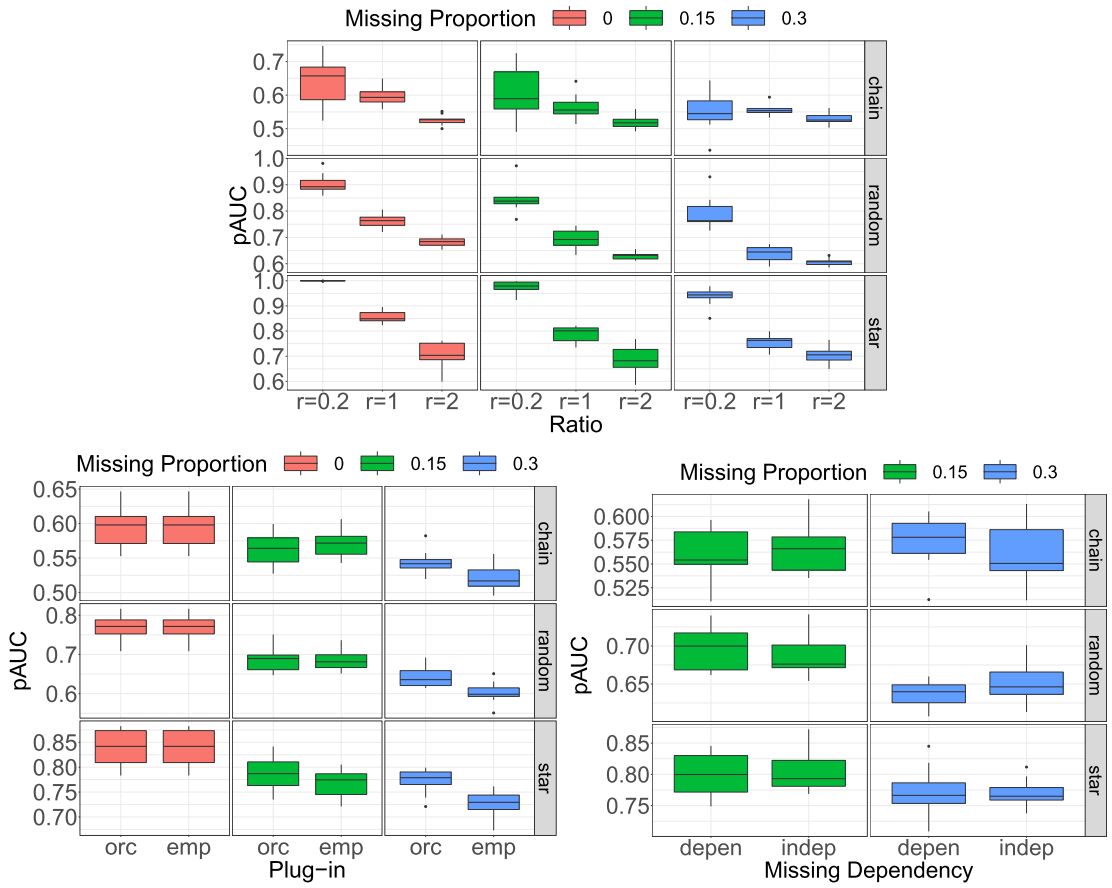

FIG 12. (Top) Boxplots of the pAUC with different ratios $r(=p / n)=0.2,1,2$. Here, $n=100$ and the dependent missing structure are assumed. The oracle IPW estimator is plugged-in. (Bottom left) Boxplots of the pAUC for support recovery with different plug-in estimators. Here, $n=100, r=2$, and the dependent missing structure are assumed. (Bottom right) Boxplots of the pAUC for support recovery with different missing structures ("depen" and "indep"). Here, $n=100$ and $r=1$ are assumed. The oracle IPW estimator is plugged-in. 

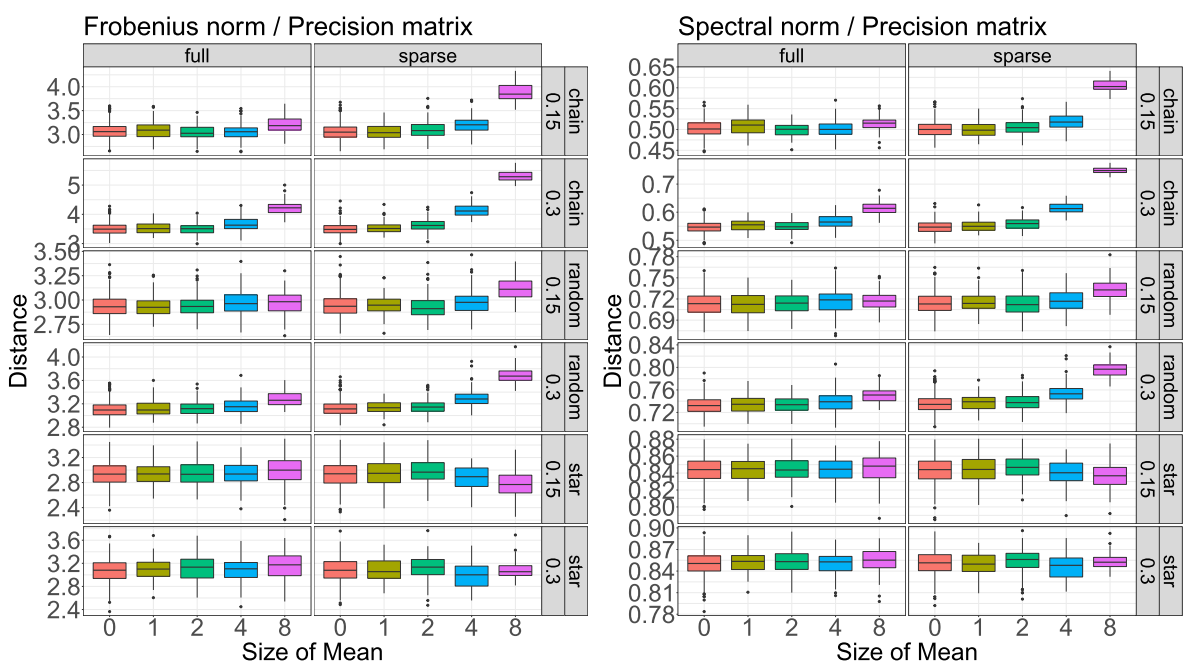

FIG 13. Boxplots of the Frobenius (left) spectral (right) norms $\|\widehat{\mathbf{\Omega}}-\mathbf{\Omega}\|$ with different magnitudes of an unknown mean vector. Here, $n=100, r=1$, and the dependent missing structure are assumed. We randomly generate 100 data sets.

log-likelihood function below;

$$
C V(\lambda)=\sum_{k=1}^{K} \sum_{i \in G_{k}}\left\{\log \left|\boldsymbol{\Omega}_{\lambda}^{(k)}\right|+X_{i}^{\mathrm{T}} \boldsymbol{\Omega}_{\lambda}^{(k)} X_{i}\right\}
$$

We let $\boldsymbol{\Omega}_{C V}=\boldsymbol{\Omega}_{\lambda_{C V}}$ the precision matrix at this level of the optimal sparsity $\lambda_{C V}$. It turns out $\lambda_{C V}$ is close to, but slightly smaller than the smallest tuning parameter $\lambda_{1}$. The four precision matrix models have 36, $170\left(\lambda_{1}\right), 5,860\left(\lambda_{2}\right)$, $14\left(\lambda_{3}\right), 35,630\left(\lambda_{C V}\right)$ non-zero elements (except diagonals) in each.

We impose missing values on the complete data matrix in a similar manner described in Section D. For this analysis, we assume the independent missing structure and note that results do not alter significantly using the dependent structure. To estimate $\boldsymbol{\Omega}_{\lambda_{i}}$, we solve the graphical lasso (13) using the incomplete data with the tuning parameter fixed at $\lambda_{i}$. Since the optimality of the tuning parameter can vary as different data is available due to missing, the crossvalidation procedure is separately performed, instead of using the same $\lambda_{C V}$ to estimate $\boldsymbol{\Omega}_{C V}$. Let $\widehat{\boldsymbol{\Omega}}_{\lambda}^{(k)}$ be the solution with the tuning parameter $\lambda$ without the $k$-th fold of incomplete data. Then, the (cross-validated) log-likelihood is computed over observed data as follows;

$$
C V_{\text {mis }}(\lambda)=\sum_{k=1}^{K} \sum_{i \in G_{k}}\left\{\log \left|\left(\boldsymbol{Q}_{i}^{(k)}\right)^{-1}\right|+X_{i, o b s}^{\mathrm{T}}\left(\boldsymbol{Q}_{i}^{(k)}\right)^{-1} X_{i, o b s}\right\}
$$

where $\boldsymbol{Q}_{i}^{(k)}=\left(\left(\widehat{\boldsymbol{\Omega}}_{\lambda}^{(k)}\right)^{-1}\right)_{i, o b s}=\left(\left(\left(\widehat{\boldsymbol{\Omega}}_{\lambda}^{(k)}\right)^{-1}\right)_{k \ell}, k, \ell \in\left\{k: \delta_{i k}=1\right\}\right)$ and $X_{i, o b s}=$ 


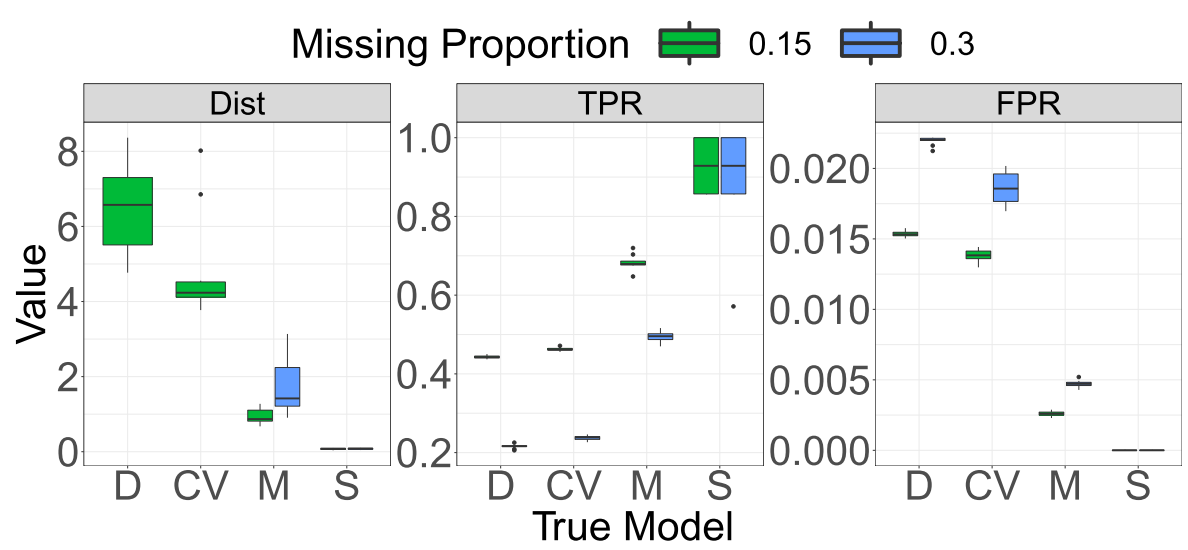

FIG 14. Boxplot of performance measures (left: the error distance, middle: TPR, right: FPR) using the riboflavin data. " $D$ ", " $M$ ", " $S$ ", and " $C V$ " on the $x$-axis stand for the dense $\left(\lambda_{1}\right)$, moderate $\left(\lambda_{2}\right)$, sparse $\left(\lambda_{3}\right)$, and cross-validated $\left(\lambda_{C V}\right)$ models, respectively. Due to readability, two boxplots for the distance from " $D$ " and " $C V$ " are not shown when the missing proportion is $30 \%$.

TABLE 2

Quantiles for the spectral norms of the dense (" $D$ ") and cross-validated ("CV") models with the missing proportion $30 \%$.

\begin{tabular}{c|c|c|c|c|c}
\hline & $\min$ & Q1 & Q2 & Q3 & $\max$ \\
\hline D & 62.135 & 771.178 & 4340.741 & 8749.103 & 16449.95 \\
\hline CV & 26.656 & 30.359 & 53.212 & 3939.772 & 34043.44 \\
\hline
\end{tabular}

$\left(X_{i k}, k \in\left\{k: \delta_{i k}=1\right\}\right)^{\mathrm{T}}$. Let $\hat{\lambda}_{C V}$ the optimal parameter that minimizes $C V_{\text {mis }}$ and $\widehat{\boldsymbol{\Omega}}_{C V}$ the graphical lasso solution using all observed data at $\hat{\lambda}_{C V}$.

Figure 14 presents three different measures to assess precision matrix estimation. An error distance between the truth and an estimate is evaluated by the spectral norm. Due to readability, the boxplots of the distance for dense models ("D" and "CV") under the missing proportion $30 \%$ are not shown, but their summary statistics are provided in Table 2. It is confirmed again that having more missing values yields worse estimation. Also, it is possible to see that the denser model is more difficult to achieve satisfactory accuracy in estimation and graph recovery.

\section{Acknowledgments}

We appreciate the two anonymous reviewers and the Associate Editor whose comments helped us improve this manuscript significantly.

\section{References}

[1] Allen, G. I. and Tibshirani, R. (2010). Transposable regularized covariance models with an application to missing data imputation. The Annals 
of Applied Statistics 4 764-790. MR2758420

[2] Banerjee, O., El Ghaoui, L. and D'Aspremont, A. (2008). Model Selection Through Sparse Maximum Likelihood Estimation for Multivariate Gaussian or Binary Data. Journal of Machine Learning Research 9 485516. MR2417243

[3] Bickel, P. J. and LevinA, E. (2008). Covariance regularization by thresholding. The Annals of Statistics 36 2577-2604. MR2485008

[4] Bickel, P. J. and Levina, E. (2008). Regularized estimation of large covariance matrices. The Annals of Statistics 36 199-227. MR2387969

[5] Cai, T., Liu, W. and Luo, X. (2011). A Constrained $\ell_{1}$ Minimization Approach to Sparse Precision Matrix Estimation. Journal of the American Statistical Association 106 594-607. MR2847973

[6] Cai, T. T., Ren, Z. and Zhou, H. H. (2016). Estimating structured highdimensional covariance and precision matrices: Optimal rates and adaptive estimation. Electronic Journal of Statistics 10 1-59. MR3466172

[7] CAI, T. T. and Zhang, A. (2016). Minimax rate-optimal estimation of high-dimensional covariance matrices with incomplete data. Journal of Multivariate Analysis 150 55-74. MR3534902

[8] Cui, R., Groot, P. and Heskes, T. (2017). Robust Estimation of Gaussian Copula Causal Structure from Mixed Data with Missing Values. In 2017 IEEE International Conference on Data Mining (ICDM) 835-840.

[9] Dai, B., Ding, S. and Wahba, G. (2013). Multivariate Bernoulli distribution. Bernoulli 19 1465-1483. MR3102559

[10] Datta, A. and Zou, H. (2017). CoCoLasso for high-dimensional error-invariables regression. Ann. Statist. 45 2400-2426. MR3737896

[11] FAn, J., LiaO, Y. and Liu, H. (2016). An overview of the estimation of large covariance and precision matrices. The Econometrics Journal 19 C1-C32. MR3501529

[12] Fan, J., Zhang, J. and Yu, K. (2012). Vast Portfolio Selection With Gross-Exposure Constraints. Journal of the American Statistical Association 107 592-606. MR2980070

[13] Friedman, J., Hastie, T. and Tibshirani, R. (2008). Sparse inverse covariance estimation with the graphical lasso. Biostatistics 9 432-441.

[14] Glanz, H. and Carvalho, L. (2018). An expectation-maximization algorithm for the matrix normal distribution with an application in remote sensing. Journal of Multivariate Analysis 167 31-48. MR3830632

[15] Greenewald, K., Park, S., Zhou, S. and Giessing, A. (2017). Timedependent spatially varying graphical models, with application to brain fMRI data analysis. In Advances in Neural Information Processing Systems 30 (I. Guyon, U. V. Luxburg, S. Bengio, H. Wallach, R. Fergus, S. Vishwanathan and R. Garnett, eds.) 5832-5840. Curran Associates, Inc.

[16] HAN, F., LU, J. and LiU, H. (2014). Robust scatter matrix estimation for high dimensional distributions with heavy tails. Technical report, Princeton University.

[17] Hastie, T., Tibshirani, R., Narasimhan, B. and Chu, G. (2018). impute: impute: Imputation for microarray data $\mathrm{R}$ package version 1.56.0. 
[18] Hsien, C.-J., Sustik, M. A., Dhillon, I. S. and Ravikumar, P. (2014). QUIC: Quadratic Approximation for Sparse Inverse Covariance Estimation. Journal of Machine Learning Research 15 2911-2947. MR3277149

[19] Huang, J. Z., Liu, L. and Liu, N. (2007). Estimation of Large Covariance Matrices of Longitudinal Data With Basis Function Approximations. Journal of Computational and Graphical Statistics 16 189-209. MR2345752

[20] Harrell JR, F. E., With contributions from Charles Dupont and MANY OTHERS. (2019). Hmisc: Harrell Miscellaneous R package version $4.2-0$.

[21] Katayama, S., Fujisawa, H. and Drton, M. (2018). Robust and sparse Gaussian graphical modelling under cell-wise contamination. Stat 7 e181. MR3796723

[22] Kim, J. K. and Shao, J. (2013). Statistical Methods for Handling Incomplete Data, 1 ed. 10. Chapman and Hall. MR3307946

[23] Kolar, M. and XIng, E. P. (2012). Estimating Sparse Precision Matrices from Data with Missing Values. In Proceedings of the 29th International Coference on International Conference on Machine Learning. ICML'12 635-642. Omnipress, USA.

[24] Liang, F., JiA, B., Xue, J., Li, Q. and Luo, Y. (2018). An imputationregularized optimization algorithm for high dimensional missing data problems and beyond. Journal of the Royal Statistical Society: Series B (Statistical Methodology) 80 899-926. MR3874303

[25] Little, R. J. A. and Rubin, D. B. (1986). Statistical Analysis with Missing Data. John Wiley \& Sons, Inc., New York, NY, USA. MR0890519

[26] Liu, H., Han, F., Yuan, M., Lafferty, J. and Wasserman, L. (2012). HIGH-DIMENSIONAL SEMIPARAMETRIC GAUSSIAN COPULA GRAPHICAL MODELS. The Annals of Statistics 40 2293-2326. MR3059084

[27] LOH, P.-L. and TAN, X. L. (2018). High-dimensional robust precision matrix estimation: Cellwise corruption under $\epsilon$-contamination. Electronic Journal of Statistics 12 1429-1467. MR3804842

[28] LoH, P.-L. and Wainwright, M. J. (2012). High-dimensional regression with noisy and missing data: Provable guarantees with nonconvexity. The Annals of Statistics 40 1637-1664. MR3015038

[29] Lounici, K. (2014). High-dimensional covariance matrix estimation with missing observations. Bernoulli 20 1029-1058. MR3217437

[30] Pang, H., Liu, H. and Vanderbei, R. (2014). The Fastclime Package for Linear Programming and Large-scale Precision Matrix Estimation in R. Journal of Machine Learning Research 15 489-493.

[31] PARK, S. and Lim, J. (2019). Non-asymptotic rate for high-dimensional covariance estimation with non-independent missing observations. Statistics E Probability Letters 153 113-123. MR3964185

[32] Pavez, E. and Ortega, A. (2018). Active Covariance Estimation by Random Sub-Sampling of Variables. In 2018 IEEE International Conference on Acoustics, Speech and Signal Processing (ICASSP) 4034-4038. 
[33] Pavez, E. and Ortega, A. (2019). Covariance Matrix Estimation with Non Uniform and Data Dependent Missing Observations. MR4232009

[34] Rao, M., Javidi, T., Eldar, Y. C. and Goldsmith, A. (2017). Estimation in autoregressive processes with partial observations. In 2017 IEEE International Conference on Acoustics, Speech and Signal Processing (ICASSP) 4212-4216.

[35] Ravikumar, P., Wainwright, M. J., Raskutti, G. and Yu, B. (2011). High-dimensional covariance estimation by minimizing $\ell_{1}$-penalized log-determinant divergence. Electronic Journal of Statistics 5 935-980. MR2836766

[36] Rohde, A. and Tsybakov, A. B. (2011). Estimation of high-dimensional low-rank matrices. The Annals of Statistics 39 887-930. MR2816342

[37] Rothman, A. J., Levina, E. and Zhu, J. (2009). Generalized Thresholding of Large Covariance Matrices. Journal of the American Statistical Association 104 177-186. MR2504372

[38] Rudelson, M. and Vershynin, R. (2013). Hanson-Wright inequality and sub-gaussian concentration. Electronic Communications in Probability $\mathbf{1 8}$ 9 pp. MR3125258

[39] Saulis, L. and StatulevičIus, V. (1991). Limit theorems for large deviations. Springer Science Business Media. MR1171883

[40] Schneider, T. (2001). Analysis of Incomplete Climate Data: Estimation of Mean Values and Covariance Matrices and Imputation of Missing Values. Journal of Climate 14 853-871.

[41] StÄDler, N. and Bühlmann, P. (2012). Missing values: sparse inverse covariance estimation and an extension to sparse regression. Statistics and Computing 22 219-235. MR2865066

[42] Thai, J., Hunter, T., Akametalu, A. K., Tomlin, C. J. and BAYEN, A. M. (2014). Inverse covariance estimation from data with missing values using the Concave-Convex Procedure. In 53rd IEEE Conference on Decision and Control 5736-5742.

[43] van Buuren, S. and Groothuis-Oudshoorn, K. (2011). mice: Multivariate Imputation by Chained Equations in R. Journal of Statistical Software 45 1-67.

[44] Vershynin, R. (2018). High-Dimensional Probability: An Introduction with Applications in Data Science. Cambridge Series in Statistical and Probabilistic Mathematics 11-37. Cambridge University Press. MR3837109

[45] Walter, S. D. (2005). The partial area under the summary ROC curve. Statistics in Medicine 24 2025-2040. MR2142723

[46] Wang, H., Fazayeli, F., Chatterjee, S. and Banerjee, A. (2014). Gaussian Copula Precision Estimation with Missing Values. In Proceedings of the Seventeenth International Conference on Artificial Intelligence and Statistics (S. KASKI and J. CorAnder, eds.). Proceedings of Machine Learning Research 33 978-986. PMLR, Reykjavik, Iceland.

[47] Xu, M. H. and Shao, H. (2012). Solving the Matrix Nearness Problem in the Maximum Norm by Applying a Projection and Contraction Method. Advances in Operations Research 2012 1-15. MR2960323 
[48] Yuan, M. (2010). High Dimensional Inverse Covariance Matrix Estimation via Linear Programming. Journal of Machine Learning Research 11 22612286. MR2719856

[49] Zhao, T., Liu, H., Roeder, K., Lafferty, J. and Wasserman, L. (2012). The Huge Package for High-dimensional Undirected Graph Estimation in R. Journal of Machine Learning Research 13 1059-1062. MR2930633

[50] Zhou, S. (2019). Sparse Hanson-Wright inequalities for subgaussian quadratic forms. Bernoulli 25 1603-1639. MR3961224 